\title{
Enhanced Coherence of a Quantum Doublet Coupled to Tomonaga-Luttinger Liquid Leads
}

\author{
Antonio Cirillo $^{\mathrm{a}}$, Matteo Mancini ${ }^{\mathrm{a}}$, Domenico Giuliano ${ }^{\mathrm{b}, *}$, Pasquale Sodano ${ }^{\mathrm{c}, \mathrm{a}}$ \\ ${ }^{a}$ Dipartimento di Fisica, Università di Perugia, Via A. Pascoli, I-06123, Perugia, Italy and \\ I.N.F.N., Sezione di Perugia, Via A. Pascoli, I-06123, Perugia, Italy \\ ${ }^{b}$ Dipartimento di Fisica, Università della Calabria Arcavacata di Rende I-87036, Cosenza, Italy and \\ I.N.F.N., Gruppo collegato di Cosenza, Arcavacata di Rende I-87036, Cosenza, Italy \\ ${ }^{c}$ Perimeter Institute for Theoretical Physics 31 Caroline St. N, Waterloo ON, N2L 2Y5, Canada
}

\begin{abstract}
We use boundary field theory to describe the phases accessible to a tetrahedral qubit coupled to Josephson junction chains acting as Tomonaga-Luttinger liquid leads. We prove that, in a pertinent range of the fabrication and control parameters, an attractive finite coupling fixed point emerges due to the geometry of the composite Josephson junction network. We show that this new stable phase is characterized by the emergence of a quantum doublet which is robust not only against the noise in the external control parameters (magnetic flux, gate voltage) but also against the decoherence induced by the coupling of the tetrahedral qubit with the superconducting leads. We provide protocols allowing to read and to manipulate the state of the emerging quantum doublet and argue that a tetrahedral Josephson junction network operating near the new finite coupling fixed point may be fabricated with today's technologies.
\end{abstract}

Keywords:

Boundary critical phenomena, Josephson junction arrays, Quantum impurity models PACS: 03.70.+k, 74.40.Kb, 74.81.Fa, 85.25.Cp

\section{Introduction}

Quantum impurity models [1] provide a natural paradigm to describe a large number of nonperturbative phenomena occurring in one dimensional quantum devices such as point contacts, constrictions, crossed quantum wires and Josephson junction (JJ) chains [2, 3, 4, 5, 6]. While a standard perturbative approach is accurate when the impurity is weakly coupled to the environmental modes needed to fully describe the quantum device, there are situations in which impurities are strongly coupled to such environmental modes: when this happens, it is impossible to disentangle the impurity from the rest of the system, the perturbative approach breaks down and one

\footnotetext{
${ }^{*}$ Corresponding author

Email addresses: antonio.cirillo@fisica.unipg.it (Antonio Cirillo), matteo.mancini@fisica.unipg.it (Matteo Mancini), domenico.giuliano@fis.unical.it (Domenico Giuliano), pasquale.sodano@pg.infn.it (Pasquale Sodano)
} 
has to resort to the non perturbative tools provided by boundary field theories (BFT) $[1,7]$, which have been shown to yield accurate descriptions of many realistic low dimensional systems [8].

Prototypical non perturbative impurities states are realized in systems exhibiting the Kondo effect $[9,10,11,12]$, or in situations where static defects appear in Tomonaga-Luttinger liquids (TLL)s; for both settings, a renormalization group approach leads, after bosonization [8], to the emergence of boundary sine-Gordon models $[13,14]$. In both cases, the interaction with the impurity makes the boundary coupling strength to scale to a stable strongly coupled fixed point (SFP) which is characterized by a fully screened spin in Kondo systems or by the effective disappearance of the impurity in a "healed" TLL $[15,16]$. More remarkable states are achieved when a finite coupling fixed point (FFP) - characterized by new non-trivial universal indices - emerges; for instance, this happens in the overscreened Kondo problems [9, 10, 11, 12], or in crossed TLLs where, as a result of the crossing, some operators turn from irrelevant to marginal, leading to correlation functions exhibiting power-law decays with nonuniversal exponents [4, 5, 17].

Quantum impurities are realizable also in superconducting Josephson devices [18, 19, 20, 21]. Superconducting Josephson chains with a weak link [18, 22] and SQUIDs [19, 23] may be indeed described by boundary sine Gordon models yielding a phase diagram with only two fixed points: an unstable weakly coupled fixed point (WFP), and a stable one at strong coupling. At variance, for pertinent architectures of the Josephson junction network (JJN) one may find a range of fabrication and control parameters where a stable FFP emerges in the phase diagram [6] allowing for the engineering of superconducting devices exhibiting enhanced quantum coherence [24] and $4 e$ superconductivity [20]. The stable fixed point is associated with the emergence of a doubly degenerate ground state which may be regarded as a quantum doublet described by a spin $1 / 2$ degree of freedom coupled to the plasmon modes via the boundary interaction; as a result, one may use superconducting devices not only as good candidates for the design of solid state quantum bits [25] but also as efficient quantum simulators of the various physical behaviors realizable in Kondo systems.

Engineering quantum doublets robust against noise and decoherence is of paramount importance for applications to quantum information processing. Quite recently a remarkably robust two-level quantum system has been shown to emerge in a device made with six JJs arranged (see Fig.1) in a symmetric tetrahedral geometry [26, 27]. In Refs.[26, 27] it has been pointed out that, when each internal loop is pierced by a dimensionless magnetic flux $f=\pi$, the ground state is doubly degenerate and that the degeneracy is robust against small variations in the applied gate voltages and/or in the applied magnetic flux, since the degeneracy is split only to second order in the charge and flux noise. Remarkably, the design of a tetrahedral quantum bit $[26,27]$ may be modified so as to make it robust also against the noise in the Josephson junction energy [28].

For any practical purpose (control or reading out the state of the quantum bit) one needs to connect the tetrahedral quantum bit to external leads, which may induce new decoherence effects spoiling the coherence of the quantum doublet [29]. As we shall show, realizing the leads with TLLs, enhances the coherence of this device. This happens if one uses one-dimensional superconducting leads which may be mapped onto TLLs with the Luttinger parameter $g$ depending on the fabrication parameters of the $\operatorname{JJN}[18,19,22,23,30,31]$. In this paper, we address this issue by analysing the device made by the central tetrahedral JJN - T - depicted in Fig. 1 connected to three JJ chains ending in bulk superconductors at fixed phase $\phi_{j}, j=1,2,3$; the resulting network 
is depicted in Fig.2. We show explicitly that the interaction of the central region $\mathbf{T}$ with the lowenergy collective excitations (plasmons) of the leads merely renormalizes the "bare" parameters of $\mathbf{T}$ and, thus, does not break the tetrahedral "symmetry" responsible for the robust groundstate degeneracy[26, 27]. As a result we exhibit a JJN which is robust also against fluctuations of the lead's parameters and argue that such network may be fabricated with nowadays technolgies [32].

The paper is organized as follows.

In section 2, we derive an effective spin-1/2 Hamiltonian for T (Fig.1). In agreement with the results of refs.[26, 27], we find that the spectrum of the effective Hamiltonian admits a twofold degenerate groundstate and argue that, in the effective theory, $\mathbf{T}$ may be regarded as a spin-1/2 degree of freedom $\mathbf{S}_{G}$. Finally, we derive the low-energy description of the JJN depicted in Fig.2. We show then that the effective theory is a $1+1$ dimensional field theory, where the central region is described by a spin interacting with the leads via a pertinent boundary interaction.

In section 3 we use a perturbative approach to account for the couplings between $\mathbf{T}$ and the TLL leads realized with JJ chains. Here, we use a renormalization group approach to determine the flow of the running boundary coupling strengths, as a function of the system size and we determine the range of values of $g$ and $f$ where the perturbative approach breaks down.

In section 4, we analyze the large-scale behavior of the JJN depicted in Fig. 2 in the strong coupling limit where the boundary effects induced by $\mathbf{T}$ become relevant. In particular, we find the set of minima of the boundary interaction potential at the SFP and construct the instanton operators connecting two degenerate minima. Using the renormalization group approach [33], we analyse the flow of the coupling constants associated to the instantons. Finally, we show that - for $-\frac{\pi}{10} \lesssim f-\pi \lesssim \frac{\pi}{10}$ and $1<g<3$ - a finite coupling fixed point emerges.

In section 5 we show that instantons are responsible for the emergence of a two-level quantum system which, due to the network's architecture, is robust, for $-\frac{\pi}{10} \lesssim f-\pi \lesssim \frac{\pi}{10}$, against the decoherence arising from coupling the cental region to the leads. Moreover, we show how the state of the emerging quantum doublet may be manipulated by acting upon the external control parameters.

In section 6 we point out that the quantum doublet emerging in a tetrahedral JJN operating near the FFP may be realized with today's technologies.

Section 7 is devoted to concluding remarks.

The appendices provide the necessary mathematical background to follow the analysis carried in the text.

\section{Boundary field theory description of the tetrahedral JJN}

In this section, we derive the low-energy, long-wavelength description of the JJN depicted in Fig.2. We shall find that this JJN may be described by a $1+1$ dimensional field theory, with a pertinent boundary interaction describing the central region. In the following we shall firstly derive the Hamiltonian associated to the central region $\mathbf{T}$ and then construct the BFT describing the JJN in which $\mathbf{T}$ is connected to TLL leads realized with JJ chains ending with three bulk superconductors held at fixed phases $\varphi_{1}, \varphi_{2}, \varphi_{3}$. 


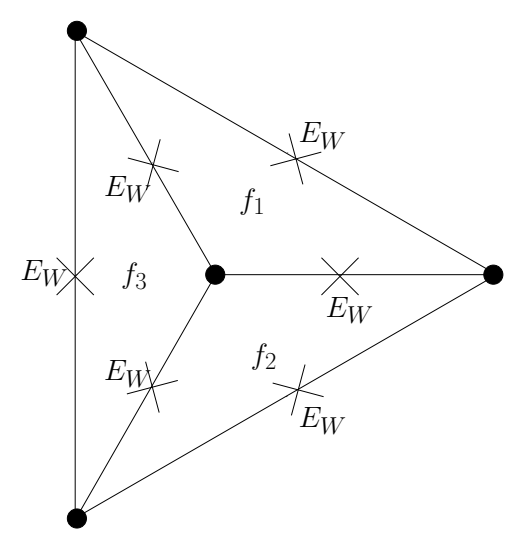

Figure 1: The central region $\mathbf{T}$. The junctions are assumed to have the same Josephson energy $E_{W}$ while the three loops are threaded by the dimensionless fluxes $f_{1}, f_{2}, f_{3}$, respectively.

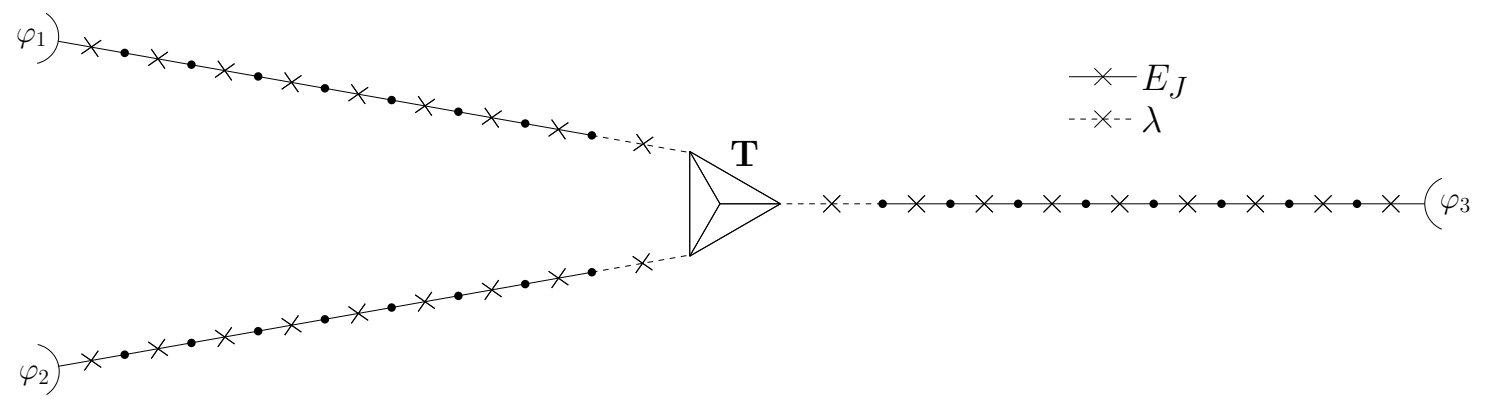

Figure 2: The tetrahedral JJN. The central region T, depicted in Fig.1, is made with four superconducting grains connected by six quantum Josephson junctions tuned nearby the degeneracy between two charge eigenstates. $\mathbf{T}$ is connected, via a coupling $\lambda \ll E_{W}$, to three leads realized with one-dimensional Josephson junction arrays fabricated with junctions of nominal Josephson energy $E_{J}$ and ending in three bulk superconductors held at fixed phases $\varphi_{1}, \varphi_{2}, \varphi_{3}$

\subsection{The central region $\mathbf{T}$}

T is fabricated with six quantum Josephson junction, joined to each other as depicted in Fig.1. We assume that, for each junction, the charging energy $E_{C}$ is much bigger than the Josephson energy $E_{W}$. To prevent Coulomb blockade from forbidding charge transport across $\mathbf{T}$, we further assume that to each superconducting grain is applied a gate voltage $V_{g}$, tuned nearby the degeneracy between the charge eigenstates with charge equal to $\mathcal{N}$ and $\mathcal{N}+1$. Under these assumptions one can describe each superconducting grain with a quantum spin-1/2 variable $\mathbf{S}_{0}^{(i)}[18,19,21]$; this leads to an effective spin-1/2 representation for the Hamiltonian $\mathcal{H}_{\mathrm{T}}$ describing the central region.

If each internal loop in Fig. 1 is pierced by a (dimensionless) magnetic flux $f$ (i.e. $f_{1}=f_{2}=$ 
$\left.f_{3}=f\right)$, a standard procedure [21] yields

$$
\begin{aligned}
\mathcal{H}_{\mathrm{T}} & =-H \sum_{i=0}^{3}\left(S_{0}^{(i)}\right)^{z}-\frac{E_{W}}{2}\left[\sum_{i=1}^{3}\left(e^{i f}\left(S_{0}^{(i)}\right)^{+}\left(S_{0}^{(i+1)}\right)^{-}+e^{-i f}\left(S_{0}^{(i+1)}\right)^{+}\left(S_{0}^{(i)}\right)^{-}\right)+\right. \\
& \left.+\sum_{i=1}^{3}\left(\left(S_{0}^{(i)}\right)^{+}\left(S_{0}^{(0)}\right)^{-}+\left(S_{0}^{(0)}\right)^{+}\left(S_{0}^{(i)}\right)^{-}\right)\right] .
\end{aligned}
$$

In Eq.(1), $\mathbf{S}_{0}^{(i)}, i=1,2,3$, denotes an effective spin-1/2 operator lying on the three outer sites of $\mathbf{T}$, while $\mathbf{S}_{0}^{(0)}$ lies on the central site. The parameter $H \propto e^{*} V_{g}-N-\frac{1}{2}$ is determined by the uniform gate voltage bias.

$\mathcal{H}_{\mathrm{T}}$ can be exactly diagonalized: its eigenvalues and eigenstates are given in Appendix A. There we show also that, for $\frac{\pi}{2}<f<\frac{3 \pi}{2}$ and $|H| \ll E_{W}$, the ground state is twofold degenerate; here, we denote the states of the degenerate quantum doublet as $|0,1\rangle \equiv|\Uparrow\rangle$ and $|0,2\rangle=|\downarrow\rangle$. The effective spin-1/2 operator $\mathbf{S}_{G}$, acting onto the two-dimensional subspace spanned by $\{|\uparrow\rangle,|\Downarrow\rangle\}$ may be then represented as

$$
\mathbf{S}_{G}^{a}=\frac{1}{2} \sum_{\sigma, \sigma^{\prime}}|\sigma\rangle\left\langle\sigma^{\prime}\right| \tau_{\sigma, \sigma^{\prime}}^{a},
$$

where $a=1 \ldots 3, \tau$ are the Pauli matrices and $\sigma=\Uparrow, \Downarrow$.

Although a spin-1/2 degree of freedom emerges quite naturally in pertinently engineered JJN $[6,20,21]$, its robustness - against any detuning of $e^{*} V_{g}$ off the degeneracy value $N+\frac{1}{2}$ as well as against any small deviation of the dimensionless magnetic flux $f$ from its optimal value $\pi$ is a very challenging task. Indeed in all the devices analyzed in Refs.[6, 20, 21] the twofold degeneracy is realized only if $f$ is fine-tuned to $\pi$, since any displacement from $f=\pi$ breaks the doublet degeneracy already to the first order in $f-\pi$. At variance, due to the frustration induced by the presence of the central spin $\left.S_{0}^{(} 0\right)$, the tetrahedral central region $\mathbf{T}$ is much more stable against noise in the external control parameters.

\subsection{The emerging quantum doublet $\mathbf{S}_{G}$}

A simple symmetry argument provides us with an hint on why the architecture of the central region is relevant for the robustness of the emerging quantum doublet. For this porpouse we first prove that this robustness depends crucially on if the number of junctions needed to fabricate the central region is even or odd.

If the central region is realized with an odd number of junctions -as it happens, for instance, in the Y-shaped networks analyzed in [6]- the ground state degeneracy is a consequence of the invariance of the effective Hamiltonian under time-reversal symmetry $\hat{T}$ which, for a three-spin effective Hamiltonian describing a triangle-shaped central region [6], is explicitly realized as

$$
\hat{T}=\left[\prod_{j=1}^{3} \sigma_{j}^{x}\right] \hat{T}_{123},
$$


where the operator $\left[\prod_{j=1}^{3} \sigma_{j}^{x}\right]$ ( $\sigma^{x}$ being the first Pauli matrix) changes the sign of each one of the three spins, while $\hat{T}_{123}$ reverses the label order of the spins (that is, $(123) \longrightarrow(321)^{1}$ ). Since, when the number of spins is odd, the ground state has total spin $1 / 2$, and since $\hat{T}$ reverses the sign of the total spin, one sees immediately that the ground state must be twofold degenerate. Such a degeneracy is, however, easily spoiled by a displacement of the applied gate voltage off the degeneracy point, i.e., by a nonzero value of the parameter $H$, which is coupled linearly to the total spin of the state: any detuning of $e^{*} V_{g}$ off the degenerate value $N+\frac{1}{2}$ breaks then the ground state degeneracy already to the first order in $e^{*} V_{g}-N-\frac{1}{2}$. At variance, when the central region is effectively described by a spin Hamiltonian with an even number of spins (i.e., the number of junctions needed to fabricate the central region is even) the ground state is a spin singlet and, thus, insensitive to a nonzero value of $H$. Although robustness versus accidental displacements in the applied gate voltage is guaranteed, to get a degenerate ground state requires a fine tuning of the applied flux $f$ to $f=\pi$ : any displacement of the applied flux off the optimal value $f-\pi$ breaks the degeneracy between the two ground states already to the first order in $f-\pi$.

The tetrahedral geometry of the central region $\mathcal{H}_{\mathrm{T}}$ provides an optimal compromise between the above complementary issues, since the twofold ground state degeneracy is protected by symmetries that are realized for any value of $f$. Indeed, time-reversal $\hat{T}$ is now realized as $\mathbf{S}_{s}=\mathbf{I}_{S} \hat{T}_{123}$ where $\mathbf{I}_{S}$ reverses the sign of all the spins and $\hat{T}_{123}$ is defined in Eq.(3). Looking at the explicit form of the states $|\Uparrow\rangle,|\Downarrow\rangle$, given in appendix Appendix A, one may readily see that

$$
|\Downarrow\rangle=\mathbf{I}_{S}|\Uparrow\rangle, \quad|\Uparrow\rangle=\mathbf{I}_{S}|\Downarrow\rangle \quad,
$$

and that

$$
\hat{T}_{123}|\Uparrow\rangle=e^{-\frac{2 i \pi}{3}}|\Uparrow\rangle, \quad \hat{T}_{123}|\Downarrow\rangle=e^{\frac{2 i \pi}{3}}|\Downarrow\rangle .
$$

Since $\mathbf{I}_{S}$ is not a symmetry of $\mathcal{H}_{\mathbf{T}}$, one concludes that $\mathbf{I}_{S}|\Uparrow\rangle$ must be an eigenstate of $\mathcal{H}_{\mathrm{T}}$, independent of $|\Uparrow\rangle$, but degenerate in energy with it, as explicitly shown in Appendix A. This ensures that the twofold ground state degeneracy is allowed within a rather large range of values for the applied flux $f$.

Robustness against variations in the external flux may be easily seen also with the help of a perturbative analysis.Indeed, in order to break the degeneracy between $|\Uparrow\rangle,|\Downarrow\rangle$ one may look at situation where the three fluxes are split according to $f_{j} \rightarrow f+\delta_{j}$ (with $\left|\delta_{j} / f\right| \ll 1$ ). Writing $\mathcal{H}_{\mathrm{T}}\left[\left\{f+\delta_{i}\right\}\right]$ as

$$
\mathcal{H}_{\mathrm{T}}\left[\left\{f+\delta_{i}\right\}\right] \approx \mathcal{H}_{\mathrm{T}}[\{f\}]-i \frac{E_{W}}{2} e^{i f} \sum_{i=1}^{3}\left[\delta_{i}\left(S_{0}^{(i)}\right)^{+}\left(S_{0}^{(i+1)}\right)^{-}\right]+\text {h.c. }+O\left[\left\{\delta_{i}^{2}\right\}\right]
$$

one may easily compute matrix elements of $\mathcal{H}_{\mathrm{T}}\left[\left\{f+\delta_{i}\right\}\right]-\mathcal{H}_{\mathbf{T}}[\{f\}]$ within the subspace spanned

\footnotetext{
${ }^{1}$ Notice that multiplication of $\left[\prod_{j=1}^{3} \sigma_{j}^{x}\right]$ by $\hat{T}_{123}$ is needed in order for the symmetry to be preserved also when a magnetic flux is applied
} 
by $|\Uparrow\rangle,|\Downarrow\rangle$, provided one knows that:

$$
\begin{aligned}
& \left\langle\sigma\left|\sum_{i=1}^{3}\left[\delta_{i}\left(S_{0}^{(i)}\right)^{+}\left(S_{0}^{(i+1)}\right)^{-}\right]\right| \sigma\right\rangle \propto \sin (f) \sum_{i=1}^{3} \delta_{i} \\
& \left\langle\sigma\left|\sum_{i=1}^{3}\left[\delta_{i}\left(S_{0}^{(i)}\right)^{+}\left(S_{0}^{(i+1)}\right)^{-}\right]\right| \bar{\sigma}\right\rangle \propto \sin (f)\left(e^{i \frac{\pi}{3}} \delta_{1}-\delta_{2}+e^{-i \frac{\pi}{3}} \delta_{3}\right),
\end{aligned}
$$

with $\sigma=\Uparrow$, $\Downarrow$, and $\Uparrow=\Downarrow, \bar{\Downarrow}=\Uparrow$. From Eqs.(7), one sees that, for $f=\pi$, the ground state stays degenerate at least up to first order in the displacements $\left\{\delta_{j}\right\} \delta_{j}$. To second order in the $\left\{\delta_{j}\right\}$, one finds that the low energy eigenstates $\left|\Uparrow^{\prime}\right\rangle$ and $\left|\Downarrow^{\prime}\right\rangle$ and their corresponding eigenvalues are given by:

$$
\begin{aligned}
\left|\Uparrow^{\prime}\right\rangle & =\frac{1}{\sqrt{2}}\left(|\Uparrow\rangle+e^{i \xi}|\Downarrow\rangle\right) \\
\left|\Downarrow^{\prime}\right\rangle & =\frac{1}{\sqrt{2}}\left(|\Uparrow\rangle-e^{i \xi}|\Downarrow\rangle\right) \\
\varepsilon_{\Uparrow^{\prime}} & =-E_{W}\left(1+\frac{\delta_{1}^{2}+\delta_{2}^{2}+\delta_{3}^{2}}{12}\right)+\Delta \\
\varepsilon_{\Downarrow^{\prime}} & =-E_{W}\left(1+\frac{\delta_{1}^{2}+\delta_{2}^{2}+\delta_{3}^{2}}{12}\right)-\Delta,
\end{aligned}
$$

where

$$
\begin{aligned}
e^{i \xi} & =\frac{e^{i \frac{2 \pi}{3}} \delta_{1}^{2}+\delta_{2}^{2}+e^{-i \frac{2 \pi}{3}} \delta_{3}^{2}}{\sqrt{\left(\delta_{1}^{2}-\delta_{2}^{2}\right)+\left(\delta_{2}^{2}-\delta_{3}^{2}\right)+\left(\delta_{3}^{2}-\delta_{1}^{2}\right)}} \\
\Delta & =\frac{E_{W}}{12} \sqrt{2} \sqrt{\left(\delta_{1}^{2}-\delta_{2}^{2}\right)^{2}+\left(\delta_{2}^{2}-\delta_{3}^{2}\right)^{2}+\left(\delta_{3}^{2}-\delta_{1}^{2}\right)^{2}} .
\end{aligned}
$$

From Eq. $(8,9)$ one sees that the second order corrections to $\mathcal{H}[\{f\}]$ may be recasted into an effective Hamiltonian $\mathcal{H}_{\perp}$, describing the central region as a transverse magnetic field term, given by

$$
\mathcal{H}_{\perp}=B_{\perp} \cos (\xi) \mathbf{S}_{G}^{x}+B_{\perp} \sin (\xi) \mathbf{S}_{G}^{y} \quad, \quad\left(B_{\perp}=-\Delta\right) .
$$

Of course, when all the $\delta_{j}$ are equal then $B_{\perp}=0$.

In the next section we shall derive the BFT describing the central region $\mathbf{T}$ connected to three TLL leads realized with three JJ chains fabricated with junctions of nominal Josephson energy $E_{J}$ and ending in three bulk superconductors held at fixed phases $\varphi_{1}, \varphi_{2}, \varphi_{3}$.

\subsection{Connecting $\mathbf{T}$ to TLL leads: the boundary Hamiltonian.}

To derive the BFT describing the tetrahedral JJN depicted in Fig.2 we require at first that the leads are realized by one-dimensional JJN for which the Josephson energy $E_{J}$ is bigger than the charging energy $E_{C}$; we further assume that there is an uniform gate voltage $V_{g}$ acting on each 
junction tuned nearby the degeneracy point between the states with $\mathcal{N}$, or $\mathcal{N}+1$ Cooper pairs at each junction. As a result [18], each lead may be described by a one-dimentional spin-1/2 chain; if each chain is made out of $L$ sites, the Hamiltonian describing the leads is given by

$$
\mathcal{H}_{\text {Leads }}=-\frac{E_{J}}{2} \sum_{a=1,2,3} \sum_{j=0, L-1}\left\{\sigma_{a, j}^{+} \sigma_{a, j+1}^{-}+\sigma_{a, j+1}^{+} \sigma_{a, j}^{-}\right\}+E^{z} \sum_{a=1,2,3} \sum_{j=0}^{L-1} \sigma_{a, j}^{z} \sigma_{a, j+1}^{z}
$$

with

$$
\begin{aligned}
\sigma_{a, j}^{+} & =\mathbf{P}_{G} e^{i \phi_{a, j}} \mathbf{P}_{G}^{\dagger} \\
\sigma_{a, j}^{z} & =\mathbf{P}_{G}\left[-i \frac{\partial}{\partial \phi_{a, j}}-V_{g}\right] \mathbf{P}_{G}^{\dagger} .
\end{aligned}
$$

Here, $\phi_{a, j}$ is the is the phase of the superconducting order parameter at site- $j$ of the $a$-chain, $\mathbf{P}_{G}$ is the projector onto the subspace of the Hilbert space with either $\mathcal{N}$, or $\mathcal{N}+1$ Cooper pairs at each superconducting grain, and $E^{z}$ is the effective strength of the charge interaction between nearestneighboring junctions. As a result the low-energy long wavelength limit of the Hamiltonian (11) can be described by a one-dimensional spinless TLL Hamiltonian, given by

$$
\mathcal{H}_{\mathrm{LL}}=\frac{g}{4 \pi} \sum_{a=1}^{3} \int_{0}^{L} d x\left[\frac{1}{u}\left(\frac{\partial \Phi_{a}}{\partial t}\right)^{2}+u\left(\frac{\partial \Phi_{a}}{\partial x}\right)^{2}\right],
$$

where $\Phi_{a}$ describes the collective plasmon modes of the leads, while the Luttinger parameters $g$ and $u$ are given by $g=\frac{\pi}{2\left(\pi-\arccos \left(\frac{\Delta}{2}\right)\right)}, u=a E_{J}\left[\frac{\pi}{2} \frac{\sqrt{1-\left(\frac{\Delta}{2}\right)^{2}}}{\arccos \left(\frac{\Delta}{2}\right)}\right]\left(\Delta=\left(E^{z}-3 E_{J}^{2} / 16 E_{c}\right) / E_{J}, a\right.$ is the lattice step) $[18,22]$.

The leads are connected to $\mathbf{T}$ by means of three Josephson junctions, of nominal strength $\lambda \ll E_{W}<E_{J}$, connecting the endpoints of the leads to the outer sites of $\mathbf{T}$. The Hamiltonian describing this interaction is given by [6]

$$
\mathcal{H}_{\lambda}=-\lambda \sum_{i=1}^{3}\left(\left(S_{0}^{(i)}\right)^{+} e^{-i \frac{\Phi_{i}(0)}{\sqrt{2}}}+\left(S_{0}^{(i)}\right)^{-} e^{i \frac{\Phi_{i}(0)}{\sqrt{2}}}\right) .
$$

Using Eq.(15) together with the spectrum of $\mathbf{T}$ given in Appendix A, the Schrieffer-Wolff (SW) procedure yields an effective boundary Hamiltonian involving only the low-energy degrees of freedom of $\mathbf{T}$. A rather lengthy computation yields

$$
\begin{aligned}
\mathcal{H}_{\mathbf{B}}= & 2 E_{1} I \sum_{i} \cos \left[\frac{\Phi_{i}(0)-\Phi_{i+1}(0)}{\sqrt{2}}\right]+4 E_{z} \mathbf{S}_{G}^{z} \sum_{i} \cos \left[\frac{\Phi_{i}(0)-\Phi_{i+1}(0)}{\sqrt{2}}+\frac{\pi}{2}\right]+ \\
& +4 E_{3} \mathbf{S}_{G}^{x} \sum_{j} \cos \left[\frac{2}{3} \pi(j-2)\right] \cos \left[\frac{\Phi_{j}(0)-\Phi_{j+1}(0)}{\sqrt{2}}\right]+ \\
& +4 E_{3} \mathbf{S}_{G}^{y} \sum_{j} \sin \left[\frac{2}{3} \pi(j-2)\right] \cos \left[\frac{\Phi_{j}(0)-\Phi_{j+1}(0)}{\sqrt{2}}\right]+B_{\|} \mathbf{S}_{G}^{z},
\end{aligned}
$$


where

$$
E_{1}=\frac{E_{J} \lambda^{2}}{3\left(E_{J}^{2}-4 H^{2}\right)}, E_{z}=\frac{2 H \lambda^{2}}{\sqrt{3}\left(E_{J}^{2}-4 H^{2}\right)}, E_{3}=2 E_{1},
$$

and

$$
B_{\|}=-\frac{24 \sqrt{3} \lambda^{2} H(f-\pi)}{E_{J}^{2}} .
$$

One sees from $(16,17,18)$ that the term $B_{\|} \sigma_{G}^{z}$ explicitly breaks - to the second order in the control parameters $g$ and $f$ - the degeneracy between $|\Uparrow\rangle$ and $|\Downarrow\rangle$. In the following sections we shall show how the low-energy plasmon modes of the leads renormalize the parameters of $\mathcal{H}_{\mathbf{B}}$.

\section{Perturbative analysis near the WFP}

In this section, we determine the flow of the running boundary coupling strengths, and argue about the emergence of nonperturbative fixed point in the phase diagram accessible to the tetrahedral JJN.

\subsection{Renormalization group flow of the boundary coupling near the WFP.}

To check the stability of WFP, we derive the renormalization group (RG) equations for the running boundary coupling strengths. To do so, we use of a boundary version of the RG approach to perturbed conformal field theories, developed by Cardy [34]. The starting point is given by the Euclidean boundary action $S_{\mathbf{B}}^{(I)}$, corresponding to the Hamiltonain in Eq.(16), which is given by

$$
\begin{aligned}
S_{\mathbf{B}}^{(I)}= & 2 E_{1} \int_{0}^{\beta} d \tau \sum_{j} \cos \left(\vec{\alpha}_{j} \cdot \vec{\chi}(\tau)\right)+4 E_{z} \int_{0}^{\beta} d \tau \mathbf{S}_{G}^{z} \sum_{j} \cos \left(\vec{\alpha}_{j} \cdot \vec{\chi}(\tau)+\frac{\pi}{2}\right)+ \\
& +4 E_{3} \int_{0}^{\beta} d \tau \mathbf{S}_{G}^{x} \sum_{j} \cos \left(\frac{2}{3} \pi(j-2)\right) \cos \left(\vec{\alpha}_{j} \cdot \vec{\chi}(\tau)\right)+4 E_{3} \int_{0}^{\beta} d \tau \mathbf{S}_{G}^{y} \sum_{j} \sin \left(\frac{2}{3} \pi(j-2)\right) \cos \left(\vec{\alpha}_{j} \cdot \vec{\chi}(\tau)\right)
\end{aligned}
$$

with $\beta=\left(k_{B} T\right)^{-1}$,

$$
\vec{\alpha}_{1}=\left(\begin{array}{l}
1 \\
0
\end{array}\right) \quad, \quad \vec{\alpha}_{2}=\left(\begin{array}{c}
-1 / 2 \\
\sqrt{3} / 2
\end{array}\right) \quad, \quad \vec{\alpha}_{3}=\left(\begin{array}{c}
-1 / 2 \\
-\sqrt{3} / 2
\end{array}\right) .
$$

We have defined $\vec{\chi}(\tau)=\vec{\chi}(0, \tau)=\left[\chi_{1}(x, \tau), \chi_{2}(x, \tau)\right]$, with $\chi_{1}(x, \tau)=\frac{1}{\sqrt{2}}\left[\Phi_{1}(x, \tau)-\Phi_{2}(x, \tau)\right]$, $\chi_{2}(x, \tau)=\frac{1}{\sqrt{6}}\left[\Phi_{1}(x, \tau)+\Phi_{2}(x, \tau)-2 \Phi_{3}(x, \tau)\right]$, in order to evidence that the "center of mass" field $\Phi(x, \tau)=\frac{1}{\sqrt{3}}\left[\Phi_{1}(x, \tau)+\Phi_{2}(x, \tau)+\Phi_{3}(x, \tau)\right]$ decouples from $S_{\mathbf{B}}^{(I)}$ as expected from charge conservation at $\mathbf{T}[4,5,6]$. One may then write the "free" action $S_{0}=S_{\text {Lead }}[\vec{\chi}]+S_{\mathrm{S}}[\Phi, \Theta]$ only in term of the fields $\chi_{1}$ and $\chi_{2}$. Namely,

$$
S_{\text {Lead }}[\vec{\chi}]=\frac{g}{4 \pi} \int_{0}^{\beta} d \tau \int_{0}^{L} d x\left[\frac{1}{u}\left(\frac{\partial \vec{\chi}}{\partial \tau}\right)^{2}+u\left(\frac{\partial \vec{\chi}}{\partial x}\right)^{2}\right],
$$


and

$S_{\mathrm{S}}[\Phi, \Theta]=-\frac{i}{2} \int_{0}^{\beta} d \tau \frac{d \Phi(\tau)}{d \tau}[1-\cos (\Theta(\tau))]-\frac{1}{2} \int_{0}^{\beta} d \tau\left\{B_{\|} \cos (\Theta(\tau))+B_{\perp} \sin (\Theta(\tau)) \cos (\Phi(\tau)-\xi)\right\}$

being the imaginary time action for the quantum spin variable $\mathbf{S}_{G}$ reported in appendix Appendix $\mathrm{C}$ $(\Theta, \Phi$ are the polar angles: see appendix Appendix $C$ for details). The partition function for the $\mathrm{JJN}, \mathcal{Z}$, is then given by

$$
\mathcal{Z}=\mathcal{Z}_{0}\left\langle\mathbf{T}_{\tau} e^{-S_{\mathbf{B}}^{(I)}}\right\rangle_{(0)}
$$

where

$$
\mathcal{Z}_{0}=\int \prod_{i=1,2} \mathcal{D} \chi \int \mathcal{D} \Omega e^{-S_{\text {Lead }}[\vec{\chi}]-S_{S}[\Phi, \Theta]} .
$$

$\mathbf{T}_{\tau}$ denotes the imaginary time time-ordered product, and the boundary interaction action is written as

$$
S_{\mathbf{B}}^{(I)} \equiv \sum_{\vec{\alpha} \in \mathbb{A}} \sum_{a=x, y, z} g_{\vec{\alpha}}^{a} \int_{0}^{\beta} \frac{d \tau}{L}: e^{i \vec{\alpha} \cdot \vec{\chi}(\tau)}: \mathbf{S}_{G}^{a}(\tau)
$$

In Eq.(25) the colons : ...: denote normal ordering with respect to the vacuum of the bosonic theory [18], with all the expectation values computed with respect to the WFP Hamiltonian, and $\mathbb{A}=\left\{ \pm \vec{\alpha}_{1}, \pm \vec{\alpha}_{2}, \pm \vec{\alpha}_{3}\right\}$. Furthermore, Eq.(25) defines the dimensionless couplings $g_{\vec{\alpha}}^{a}$.

Expanding $\mathcal{Z}$ in the couplings $g_{\vec{\alpha}}^{a}$ yields

$$
\begin{aligned}
\mathcal{Z} & =\mathcal{Z}_{(0)}\left(1+\frac{1}{2} \sum_{\vec{\alpha} \in \mathbb{A}} \sum_{a, b=x, y, z} L^{-2} g_{\vec{\alpha}}^{a} g_{-\vec{\alpha}}^{b} \iint d \tau d \tau^{\prime}\left\langle: e^{i \vec{\alpha} \cdot \vec{\chi}\left(\tau^{\prime}\right)}:: e^{-i \vec{\alpha} \cdot \vec{\chi}(\tau)}:\right\rangle\left\langle\mathbf{S}_{G}^{a}\left(\tau^{\prime}\right) \mathbf{S}_{G}^{b}(\tau)\right\rangle+\right. \\
& \left.-\frac{1}{3 !} \sum_{\substack{\vec{\alpha} \cdot \vec{\beta} \cdot \vec{\gamma} \in \mathbb{A} \\
\vec{\alpha}+\vec{\beta}+\vec{\gamma}=0}} \sum_{a, b, c} L^{-3} g_{\vec{\alpha}}^{a} g_{\vec{\beta}}^{b} g_{\vec{\gamma}}^{c} \iiint d \tau d \tau^{\prime} d \tau^{\prime \prime}\left\langle: e^{i \vec{\alpha} \cdot \vec{\chi}\left(\tau^{\prime \prime}\right)}:: e^{i \vec{\beta} \cdot \vec{\chi}\left(\tau^{\prime}\right)}:: e^{i \vec{\gamma} \cdot \vec{\chi}(\tau)}:\right\rangle\left\langle\mathbf{S}_{G}^{a}\left(\tau^{\prime \prime}\right) \mathbf{S}_{G}^{b}\left(\tau^{\prime}\right) \mathbf{S}_{G}^{c}(\tau)\right\rangle+\ldots\right)
\end{aligned}
$$

where we have used the fact that $\left\langle: e^{i \vec{\alpha}^{(1)} \cdot \vec{\chi}\left(\tau^{\prime}\right)}: \ldots: e^{i \vec{\alpha}^{(n)} \cdot \vec{\chi}(\tau)}:\right\rangle$ is different from 0 only if $\sum_{j=1}^{n} \vec{\alpha}^{(j)}=0$. Following the procedure of Refs.[19, 33], one finds that a rescaling of $L$ implies a renormalization of the couplings, according to the RG flow equations that, to second order in the boundary coupling strenghts, are determined by the short-distance operator product expansions (O.P.E.s)

$$
\left\{: e^{ \pm i \vec{\alpha}_{i} \cdot \chi(\tau)}:: e^{ \pm i \vec{\alpha}_{j} \cdot \chi\left(\tau^{\prime}\right)}:\right\}_{\tau^{\prime} \rightarrow \tau^{-}} \approx\left[\frac{u\left|\tau-\tau^{\prime}\right|}{L}\right]^{-\frac{1}{g}}: e^{\mp i \vec{\alpha}_{k} \cdot \vec{\chi}(\tau)}:
$$

when $\vec{\alpha}_{i}+\vec{\alpha}_{j}+\vec{\alpha}_{k}=0$, and

$$
: e^{ \pm i \vec{\alpha}_{j} \cdot \vec{\chi}(\tau)}:: e^{\mp i \vec{\alpha}_{j} \cdot \vec{\chi}\left(\tau^{\prime}\right)}: \approx_{\tau^{\prime} \rightarrow \tau^{-}} \text {const } \pm\left|\frac{u\left(\tau-\tau^{\prime}\right)}{L}\right|^{-\frac{1}{g}}\left\{\left|\frac{u\left(\tau-\tau^{\prime}\right)}{L}\right|^{1-\frac{1}{g}} i \vec{\alpha}_{j} \cdot\left[\frac{L}{u} \frac{\partial \vec{\chi}(\tau)}{\partial \tau}\right]\right\}
$$


as well as by the O.P.E.s between the spin- $1 / 2$ operators which, may be derived starting from the equations of motion for a spin in magnetic field $B=\sqrt{B_{\|}^{2}+B_{x}^{2}+B_{y}^{2}}$, given by:

$$
\begin{aligned}
\mathbf{S}_{G}^{x}(\tau) & =\mathbf{S}_{G}^{x}(0)\left(-2 \cos ^{2} \phi \sin ^{2} \theta \sinh ^{2}\left(\frac{B \tau}{2}\right)+\cosh (B \tau)\right)+ \\
& +\mathbf{S}_{G}^{y}(0)\left(i \cos \theta \sinh (B \tau)-\sin (2 \phi) \sin ^{2} \theta \sinh ^{2}\left(\frac{B \tau}{2}\right)\right)+ \\
& -\mathbf{S}_{G}^{z}(0)\left(i \sin \theta \sin \phi \sinh (B \tau)+\sin 2 \theta \cos \phi \sinh ^{2}\left(\frac{B \tau}{2}\right)\right) \\
\mathbf{S}_{G}^{y}(\tau) & =\mathbf{S}_{G}^{y}(0)\left(-2 \sin ^{2} \phi \sin ^{2} \theta \sinh ^{2}\left(\frac{B \tau}{2}\right)+\cos ^{2} \theta \cosh (B \tau)\right)+ \\
& +\mathbf{S}_{G}^{z}(0)\left(i \cos \phi \sin \theta \sinh (B \tau)-\sin \phi \sin (2 \theta) \sinh ^{2}\left(\frac{B \tau}{2}\right)\right)+ \\
& -\mathbf{S}_{G}^{x}(0)\left(i \cos \theta \sinh (B \tau)+\sin (2 \phi) \sin { }^{2} \theta \sinh ^{2}\left(\frac{B \tau}{2}\right)\right) \\
\mathbf{S}_{G}^{z}(\tau) & =\mathbf{S}_{G}^{z}(0)\left(1+2 \sin { }^{2} \theta \sinh \left(\frac{B \tau}{2}\right)\right)+ \\
& +\mathbf{S}_{G}^{x}(0)\left(i \sin \phi \sin \theta \sinh (B \tau)-\cos \phi \sin (2 \theta) \sinh ^{2}\left(\frac{B \tau}{2}\right)\right)+ \\
& -\mathbf{S}_{G}^{y}(0)\left(i \cos \phi \sin \theta \sinh (B \tau)+\sin \phi \sin (2 \theta) \sinh ^{2}\left(\frac{B \tau}{2}\right)\right),
\end{aligned}
$$

where the angles $\phi$ and $\theta$ are defined by

$$
B_{\|}=B \cos \theta, \quad B_{x}=B \sin \theta \cos \phi, \quad B_{y}=B \sin \theta \sin \phi .
$$

The resulting O.P.E.s are:

$$
\begin{aligned}
& \left\{\mathbf{S}_{G}^{x}\left(\tau^{\prime}\right) \mathbf{S}_{G}^{x}(\tau)\right\}_{\tau^{\prime} \rightarrow \tau} \approx \frac{1}{4}+\frac{B}{2}\left(\tau^{\prime}-\tau\right)\left[\mathbf{S}_{G}^{z}(\tau) \cos \theta+\mathbf{S}_{G}^{y}(\tau) \sin \phi \sin \theta\right] \\
& \left\{\mathbf{S}_{G}^{y}\left(\tau^{\prime}\right) \mathbf{S}_{G}^{y}(\tau)\right\}_{\tau^{\prime} \rightarrow \tau} \approx \frac{1}{4}+\frac{B}{2}\left(\tau^{\prime}-\tau\right)\left[\mathbf{S}_{G}^{z}(\tau) \cos \theta+\mathbf{S}_{G}^{x}(\tau) \cos \phi \sin \theta\right] \\
& \left\{\mathbf{S}_{G}^{z}\left(\tau^{\prime}\right) \mathbf{S}_{G}^{z}(\tau)\right\}_{\tau^{\prime} \rightarrow \tau} \approx \frac{1}{4}+\frac{B}{2}\left(\tau^{\prime}-\tau\right)\left[\mathbf{S}_{G}^{z}(\tau) \cos \phi \sin \theta+\mathbf{S}_{G}^{y}(\tau) \sin \phi \sin \theta\right] \\
& \left\{\mathbf{S}_{G}^{x}\left(\tau^{\prime}\right) \mathbf{S}_{G}^{y}(\tau)\right\}_{\tau^{\prime} \rightarrow \tau} \approx \frac{i}{2} \mathbf{S}_{G}^{z}(\tau)+\frac{B}{2}\left(\tau^{\prime}-\tau\right)\left[\frac{i}{2} \mathbf{I} \cos \theta+\mathbf{S}_{G}^{x}(\tau) \sin \phi \sin \theta\right] \\
& \left\{\mathbf{S}_{G}^{y}\left(\tau^{\prime}\right) \mathbf{S}_{G}^{z}(\tau)\right\}_{\tau^{\prime} \rightarrow \tau} \approx \frac{i}{2} \mathbf{S}_{G}^{x}(\tau)+\frac{B}{2}\left(\tau^{\prime}-\tau\right)\left[\frac{i}{2} \mathbf{I} \sin \theta \cos \phi+\mathbf{S}_{G}^{y}(\tau) \cos \theta\right] \\
& \left\{\mathbf{S}_{G}^{z}\left(\tau^{\prime}\right) \mathbf{S}_{G}^{x}(\tau)\right\}_{\tau^{\prime} \rightarrow \tau} \approx \frac{i}{2} \mathbf{S}_{G}^{y}(\tau)+\frac{B}{2}\left(\tau^{\prime}-\tau\right)\left[\frac{i}{2} \mathbf{I} \sin \phi \sin \theta+\mathbf{S}_{G}^{z}(\tau) \cos \phi \sin \theta\right] \\
& \left\{\mathbf{S}_{G}^{x}\left(\tau^{\prime}\right) \mathbf{S}_{G}^{z}(\tau)\right\}_{\tau^{\prime} \rightarrow \tau} \approx-\frac{i}{2} \mathbf{S}_{G}^{y}(\tau)+\frac{B}{2}\left(\tau^{\prime}-\tau\right)\left[-\frac{i}{2} \mathbf{I} \sin \phi \sin \theta+\mathbf{S}_{G}^{x}(\tau) \cos \theta\right] \\
& \left\{\mathbf{S}_{G}^{y}\left(\tau^{\prime}\right) \mathbf{S}_{G}^{x}(\tau)\right\}_{\tau^{\prime} \rightarrow \tau} \approx-\frac{i}{2} \mathbf{S}_{G}^{z}(\tau)+\frac{B}{2}\left(\tau^{\prime}-\tau\right)\left[-\frac{i}{2} \mathbf{I} \cos \theta+\mathbf{S}_{G}^{y}(\tau) \cos \phi \sin \theta\right] \\
& \left\{\mathbf{S}_{G}^{z}\left(\tau^{\prime}\right) \mathbf{S}_{G}^{y}(\tau)\right\}_{\tau^{\prime} \rightarrow \tau} \approx-\frac{i}{2} \mathbf{S}_{G}^{x}(\tau)+\frac{B}{2}\left(\tau^{\prime}-\tau\right)\left[-\frac{i}{2} \mathbf{I} \cos \phi \sin \theta+\mathbf{S}_{G}^{z}(\tau) \sin \phi \sin \theta\right]
\end{aligned}
$$


When $B_{\|}=B_{\perp}=0$ spin operators do not evolve in imaginary time and Eq.(34a) reduces to the usual spin algebra

$$
\mathbf{S}_{G}^{a} \mathbf{S}_{G}^{b}=\frac{1}{4} \delta^{a b} \mathbf{I}+\frac{1}{2} i \epsilon^{a b c} \mathbf{S}_{G}^{c}
$$

Since at the WFP the fields $\vec{\chi}(\tau)$ and $\overrightarrow{\mathbf{S}_{G}}(\tau)$ are decoupled, from Eq. $(28,29)$ one finds that two different contributions to the boundary action are generated to the second order in the boundary coupling strenghts; to evidence these contributions, one rewrites Eq.(19) as

$$
S_{\mathbf{B}}^{(I)}=\sum_{j} \int_{0}^{\beta} d \tau\left\{\mathcal{E}_{j}\left[\mathbf{S}_{G}(\tau)\right] e^{i \vec{\alpha}_{j} \cdot \vec{\chi}(\tau)}+\overline{\mathcal{E}}_{j}\left[\mathbf{S}_{G}(\tau)\right] e^{-i \vec{\alpha}_{j} \cdot \vec{\chi}(\tau)}\right\}
$$

with the $\mathbf{S}_{G}$-dependent couplings $\mathcal{E}_{j}\left[\mathbf{S}_{G}\right]$ and $\overline{\mathcal{E}}_{j}\left[\mathbf{S}_{G}\right]$ given by

$$
\begin{aligned}
& \mathcal{E}_{j}\left[\mathbf{S}_{G}\right]=E_{1}+2 i E_{z} \mathbf{S}_{G}^{z}+2 E_{3}\left\{\cos \left[\frac{2 \pi}{3}(j-2)\right] \mathbf{S}_{G}^{x}+\sin \left[\frac{2 \pi}{3}(j-2)\right] \mathbf{S}_{G}^{y}\right\} \\
& \overline{\mathcal{E}}_{j}\left[\mathbf{S}_{G}\right]=E_{1}-2 i E_{z} \mathbf{S}_{G}^{z}+2 E_{3}\left\{\cos \left[\frac{2 \pi}{3}(j-2)\right] \mathbf{S}_{G}^{x}+\sin \left[\frac{2 \pi}{3}(j-2)\right] \mathbf{S}_{G}^{y}\right\}
\end{aligned}
$$

$\mathcal{E}_{j}\left[\mathbf{S}_{G}\right]$ and $\overline{\mathcal{E}}_{j}\left[\mathbf{S}_{G}\right]$ contribute to the boundary action in Eq.(25) through the O.P.E. as

$$
\begin{aligned}
& \sum_{j, j^{\prime}} \overline{\mathcal{E}}_{j}\left[\mathbf{S}_{G}(\tau)\right]: e^{-i \vec{\alpha}_{j} \cdot \vec{\chi}(\tau)}: \mathcal{E}_{j^{\prime}}\left[\mathbf{S}_{G}\left(\tau^{\prime}\right)\right]: e^{-i \vec{\alpha}_{j^{\prime}} \cdot \vec{\chi}\left(\tau^{\prime}\right)}: \approx_{\tau^{\prime} \rightarrow \tau^{-}}\left|\frac{u\left(\tau-\tau^{\prime}\right)}{L}\right|^{-\frac{1}{g}} \sum_{k}\left\{\left[E_{1}^{2}-4 E_{z}^{2}-2 E_{3}^{2}\right.\right. \\
& \left.\left.-4 i E_{z} E_{1} \mathbf{S}_{G}^{z}(\tau)-2 E_{1} E_{3}\left(\cos \left[\frac{2 \pi}{3}(k-2)\right] \mathbf{S}_{G}^{x}(\tau)+\sin \left[\frac{2 \pi}{3}(k-2)\right] \mathbf{S}_{G}^{y}(\tau)\right)\right]: e^{i \vec{\alpha}_{k} \cdot \vec{\chi}(\tau)}:\right\}
\end{aligned}
$$

and

$$
\begin{aligned}
& \sum_{j}\left\{\mathcal{E}_{j}\left[\mathbf{S}_{G}(\tau)\right]: e^{i \overrightarrow{\hat{\alpha}_{j}} \cdot \vec{\chi}(\tau)}: \overline{\mathcal{E}}_{j}\left[\mathbf{S}_{G}\left(\tau^{\prime}\right)\right]: e^{-i \vec{\alpha}_{j} \cdot \vec{\chi}\left(\tau^{\prime}\right)}:+\overline{\mathcal{E}}_{j}\left[\mathbf{S}_{G}(\tau)\right]: e^{-i \vec{\alpha}_{j} \cdot \vec{\chi}(\tau)}: \mathcal{E}_{j}\left[\mathbf{S}_{G}\left(\tau^{\prime}\right)\right]: e^{i \overrightarrow{\alpha_{j}} \cdot \vec{\chi}\left(\tau^{\prime}\right)}:\right\} \approx_{\tau^{\prime} \rightarrow \tau^{-}} \\
& \quad-\left|\frac{u\left(\tau-\tau^{\prime}\right)}{L}\right|^{1-\frac{2}{g}} \sum_{j}\left\{4 E_{z} E_{3}\left[\cos \left[\frac{2 \pi}{3}(j-2)\right] \mathbf{S}_{G}^{y}-\sin \left[\frac{2 \pi}{3}(j-2)\right] \mathbf{S}_{G}^{x}\right] i \vec{\alpha}_{j} \cdot\left[\frac{L}{u} \frac{\partial \vec{\chi}(\tau)}{\partial \tau}\right]\right\}
\end{aligned}
$$

From Eq.(38), one sees that, though terms proportional to $\frac{\partial \vec{\chi}}{\partial \tau}$ are not present in Eq.(19), they are dynamically generated by the renormalization group procedure, yielding a new term given by

$$
\delta S_{\mathbf{B}}^{(I)}=i \Lambda \sum_{j} \int_{0}^{\beta} d \tau\left\{\cos \left[\frac{2 \pi}{3}(j-2)\right] \mathbf{S}_{G}^{y}(\tau)-\sin \left[\frac{2 \pi}{3}(j-2)\right] \mathbf{S}_{G}^{x}(\tau)\right\} \vec{\alpha}_{j} \cdot\left[\frac{L}{u} \frac{\partial \vec{\chi}(\tau)}{\partial \tau}\right]
$$

The boundary coupling $\Lambda$ is renormalized from the terms in Eq.(38). 
For a set of (dimensionless) boundary couplings $g_{\alpha}^{a}$, the RG equations to the second order in the couplings are given by:

$$
\frac{d g_{\alpha}^{a}(l)}{d l}=\left(1-h_{\alpha}\right) g_{\alpha}^{a}(l)+\sum_{\beta, \gamma} \sum_{b, c=x, y, z} C_{\beta, \gamma, \alpha}^{b, c, a} g_{\beta}^{b}(l) g_{\gamma}^{c}(l)
$$

where $l=\ln \left(L / L_{0}\right), L_{0}$ is a reference length scale, $h_{\alpha}$ is the scaling dimension of the boundary operator associated to the coupling $g_{\alpha}^{a}$ and the coefficient $C_{\beta, \gamma, \alpha}^{b, c, a}$ are defined by the O.P.E.s. Thus, deriving the coefficients $C_{\beta, \gamma, \alpha}^{b, c, a}$ from Eqs. $(37,38)$ and taking into account that $h=\frac{1}{g}(h=1)$ for any vertex operator $e^{ \pm i \vec{\alpha}_{j} \cdot \vec{\chi}(\tau)}$ (for $\frac{\partial \chi_{1}(\tau)}{\partial \tau}, \frac{\partial \chi_{2}(\tau)}{\partial \tau}$ ), one finds that the RG equations for the dimensionless couplings $G_{1}(L)=L^{1-\frac{1}{g}} E_{1}, G_{z}(L)=L^{1-\frac{1}{g}} E_{z}, G_{3}(L)=L^{1-\frac{1}{g}} E_{3}, G_{\Lambda}(L)=\Lambda$, are given by

$$
\begin{aligned}
& \left.\frac{d G_{1}(l)}{d l}=\left[1-\frac{1}{g}\right] G_{1}(l)-2\left[G_{1}^{2}(l)-G_{z}^{2}(l)-\frac{G_{3}^{2}(l)}{2}\right] \equiv \beta_{1}\left(G_{1}, G_{z}, G_{3}, G_{\Lambda}\right)\right) \\
& \frac{d G_{z}(l)}{d l}=\left[1-\frac{1}{g}\right] G_{z}(l)+4 G_{1}(l) G_{z}(l)+2 G_{3}(l) G_{\Lambda}(l) \equiv \beta_{z}\left(G_{1}, G_{z}, G_{3}, G_{\Lambda}\right) \\
& \frac{d G_{3}(l)}{d l}=\left[1-\frac{1}{g}\right] G_{3}(l)+2 G_{1}(l) G_{3}(l)+2 G_{z}(l) G_{\Lambda}(l) \equiv \beta_{3}\left(G_{1}, G_{z}, G_{3}, G_{\Lambda}\right) \\
& \frac{d G_{\Lambda}(l)}{d l}=4 G_{z}(l) G_{3}(l) \equiv \beta_{\Lambda}\left(G_{1}, G_{z}, G_{3}, G_{\Lambda}\right) .
\end{aligned}
$$

From Eqs.(41) one sees that no linear term appears in the $\beta$-function for the running coupling $G_{\Lambda}$, since the corresponding boundary operator has scaling dimension 1 , while the ones containing the vertex operators have scaling dimension $1 / g$. Furthemore, looking at the linear term in the $\beta$-functions in Eqs.(41), one sees that the couplings $G_{1}(l), G_{z}(l), G_{3}(l)$ are all irrelevant for $g<1$ and that $G_{\Lambda}(l)$ scales roughly like $e^{\left(2-\frac{2}{g}\right) l}$, so that, for $g<1$, the term $\propto \frac{\partial \overrightarrow{\mathcal{X}}(\tau)}{\partial \tau}$ is irrelevant, as well. At variance, for $g>1, G_{1}(l), G_{z}(l), G_{3}(l)$ are all relevant, while $G_{\Lambda}(l)$ is only marginally relevant. As a result, $\delta S_{\mathbf{B}}^{(I)}$ is subleading with respect to $S_{\mathbf{B}}^{(I)}$, and, for $g>1$, one may write Eqs.(41) by keeping only leading contributions to the boundary interaction; thus one gets

$$
\begin{aligned}
& \frac{d G_{1}(l)}{d l}=\left[1-\frac{1}{g}\right] G_{1}(l)-2\left[G_{1}^{2}(l)-G_{z}^{2}(l)-\frac{G_{3}^{2}(l)}{2}\right] \equiv \beta_{1}\left(G_{1}, G_{z}, G_{3}\right) \\
& \frac{d G_{z}(l)}{d l}=\left[1-\frac{1}{g}\right] G_{z}(l)+4 G_{1}(l) G_{z}(l) \equiv \beta_{z}\left(G_{1}, G_{z}, G_{3}\right) \\
& \frac{d G_{3}(l)}{d l}=\left[1-\frac{1}{g}\right] G_{3}(l)+2 G_{1}(l) G_{3}(l) \equiv \beta_{3}\left(G_{1}, G_{z}, G_{3}\right) .
\end{aligned}
$$

From Eqs.(42), one sees that, when $g>1$, the boundary interaction provides a relevant perturbation and that the pertubative approach breaks down when the leads reach the healing length $L_{*}$, for which the biggest dimensionless coupling is of order $L_{0}^{1-\frac{1}{g}} E_{J} . L_{0}$ is the reference length such that 
$G_{i}(l=0)=L_{0}^{1-\frac{1}{g}} E_{i}$, with the $E_{i}$ 's defined in Eq.(17); since Eq.(17) shows that the biggest coupling is $G_{3}(l), L_{*}$ should be determined from the requirement that $G_{3}\left[\ln \left(\frac{L_{*}}{L_{0}}\right)\right] \sim L_{0}^{1-\frac{1}{g}} E_{J}$ yielding

$$
L_{*} \sim\left(\frac{E_{J}}{E_{3}}\right)^{\frac{g}{g-1}} L_{0} .
$$

It should be noticed that, for $g=1$, one should use Eqs.(41), instead of Eqs.(42), as all the four couplings $G_{1}(l), G_{z}(l), G_{3}(l), G_{\Lambda}(l)$ correspond to marginally relevant perturbations. To spell out the RG flows for $g=1$, one should notice that $G_{z}\left(L_{0}\right)=0$ if $H=0$; from Eqs.(41), then, the RG flows run along the manifold in parameter space defined by $G_{z}(l)=G_{\Lambda}(l)=0$ and by $G_{3}(l)=2 G_{1}(l)$. Since $G_{3}$ depends on $l$ as

$$
\frac{d G_{3}(l)}{d l}=\left[G_{3}(l)\right]^{2}
$$

one sees that the RG equations in Eq.(41) coincide with the ones obtained for the boundary coupling in the isotropic Kondo model. Thus, for $g=1$ and $G_{z}\left(L_{0}\right)=0$, the tetrahedral JJN simulates a Kondo spin with isotropic couplings to the electrons and $L_{*}$ coincides with the Kondo length $L_{K}$, given by $L_{K} \sim L_{0} e^{\frac{1}{G_{3}\left(L_{0}\right)}}$. A small value of $H$ should just slightly change the RG flow, possibly to the one corresponding to a Kondo spin with anisotropic couplings to the band electrons [35].

In the next section, starting from Eqs.(41) we characterize the SFP and investigate its stability against the leading boundary perturbation.

\section{The Strong Coupled Fixed Point}

From Eq.(41) one sees that all the boundary couplings become relevant when $g>1$; this implies (43) that, as soon as $L \geq L_{*}$, the running boundary couplings cross over towards strong coupling. At the SFP, the plasmon fields $\vec{\chi}(0)$ satisfy Dirichlet boundary conditions since their values must coincide with a minimum of $\mathcal{H}_{\mathbf{B}}$; in addition, the leading boundary interaction is a combination of phase slip (instanton) operators describing tunnelling events between the neighboring minima of $\mathcal{H}_{\mathbf{B}}$.

\subsection{Minima of $\mathcal{H}_{\mathbf{B}}$}

Dirichlet boundary conditions are set by requiring that $\chi_{1}(0), \chi_{2}(0)$ take values corresponding to a minimum of $\mathcal{H}_{\mathrm{B}}$. To determine the set of minima of $\mathcal{H}_{\mathbf{B}}$ it is most convenient to represent $[4,5]$ the spin-1/2 operator $\mathbf{S}_{G}$ as a $2 \times 2$ matrix and rewrite $\mathcal{H}_{\mathbf{B}}$ as

$$
\mathcal{H}_{\mathbf{B}}=\left(\begin{array}{cc}
V_{1}(\vec{\chi}(0))+V_{z}(\vec{\chi}(0))+B_{\|}(f) & V_{x}(\vec{\chi}(0))-i V_{y}(\vec{\chi}(0)) \\
V_{x}(\vec{\chi}(0))+i V_{y}(\vec{\chi}(0)) & V_{1}(\vec{\chi}(0))-V_{z}(\vec{\chi}(0))-B_{\|}(f)
\end{array}\right) .
$$

In Eq.(45) $B_{\|}(f)$ is given by Eq.(18) while, by comparison with Eq.(16), the $V_{i}(\vec{\chi}(0))$ 's are determined as 


$$
\begin{aligned}
& V_{1}[\vec{\chi}]=2 \bar{E}_{1} \sum_{j} \cos \left[\vec{\alpha}_{j} \cdot \vec{\chi}\right] \\
& V_{x}[\vec{\chi}]=2 \bar{E}_{3} \sum_{j} \cos \left[\frac{2 \pi}{3}(j-2)\right] \cos \left[\vec{\alpha}_{j} \cdot \vec{\chi}\right] \\
& V_{y}[\vec{\chi}]=2 \bar{E}_{3} \sum_{j} \sin \left[\frac{2 \pi}{3}(j-2)\right] \cos \left[\vec{\alpha}_{j} \cdot \vec{\chi}\right] \\
& V_{z}[\vec{\chi}]=-2 \bar{E}_{z} \sum_{j} \sin \left[\vec{\alpha}_{j} \cdot \vec{\chi}\right] .
\end{aligned}
$$

One may now easily look for the eigenvalues of $\mathcal{H}_{\mathbf{B}}$ as a function of $\chi_{1}(0)$ and $\chi_{2}(0)$ obtaining

$$
\begin{aligned}
& \Lambda_{1}(\vec{\chi}(0))=V_{1}-\sqrt{V_{x}^{2}+V_{y}^{2}+\left(V_{z}+B_{\|}\right)^{2}} \\
& \Lambda_{2}(\vec{\chi}(0))=V_{1}+\sqrt{V_{x}^{2}+V_{y}^{2}+\left(V_{z}+B_{\|}\right)^{2}} .
\end{aligned}
$$

Since, for any value of $\vec{\chi}$, one has that $\Lambda_{1} \leq \Lambda_{2}$ the minima of $\mathcal{H}_{\mathbf{B}}$ are obtained from the minima

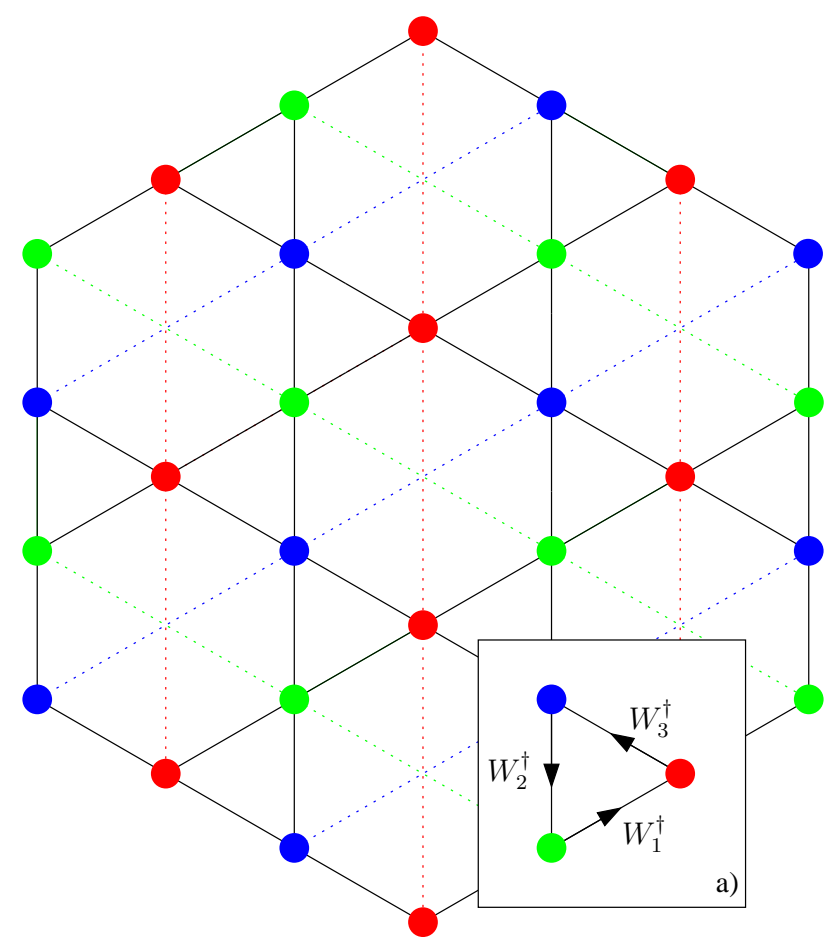

Figure 3: Lattice of the minima of $\mathcal{H}_{\mathbf{B}}$. In the box: the triangle whose vertices may be made degenerate with a pertinent choice of the external phases, and the instanton trajectories associated to the operators $W_{i}^{\dagger}$.

of $\Lambda_{1}(\vec{\chi}(0))$ only. We find that, in the $\chi_{1}(0), \chi_{2}(0)$-plane, these minima are located at the vertices 
of the Kagome lattice displayed in Fig.3. The polarization of $\mathbf{S}_{G}$ at each minimum is determined by minimizing the boundary energy after the $V_{i}$ 's have been evaluated on the pertinent vertex of the Kagome lattice of minima.

Since at the outer boundary $(x=L) \chi_{1}, \chi_{2}$ are connected to three bulk superconductors at fixed phases, the corresponding boundary conditions are given by $\chi_{1}(L, \tau)=\mu_{1}, \chi_{2}(L, \tau)=\mu_{2}$, with the relative phases $\mu_{1}, \mu_{2}$ being defined as $\mu_{1}=\left(\varphi_{1}-\varphi_{2}\right) / \sqrt{2}$, and $\mu_{2}=\left(\varphi_{1}+\varphi_{2}-2 \varphi_{3}\right) / \sqrt{6}$. Upon imposing Dirichlet boundary conditions for $\vec{\chi}(x, \tau)$ at both boundaries the mode expansion of the plasmon fields is given by

$$
\chi_{j}(x, \tau)=\xi_{j}+\sqrt{\frac{2}{g}}\left\{(L-x) \frac{\pi}{L} P_{j}-\sum_{n \neq 0} \sin \left(\frac{\pi n x}{L}\right) \frac{\alpha_{n}^{j}}{n} e^{-\frac{\pi}{L} n u \tau}\right\},
$$

where $\xi_{j}=\chi_{j}(L)$ and $P_{j}$ s are the zero mode operators.

The eigenvalues of $P_{j}$ are fixed by the boundary conditions since $\sqrt{\frac{2}{g}} \pi P_{j}=\chi_{j}(0)-\xi_{j}$; they are given by

$$
\left(p_{1}, p_{2}\right)_{l}=\sqrt{2 g}\left(-\frac{\mu_{1}}{2 \pi}+n_{12}+\epsilon_{l},-\frac{\mu_{2}}{2 \pi}+\frac{1}{\sqrt{3}}\left(2 n_{13}-n_{12}+\delta_{l}\right)\right),
$$

$n_{12}, n_{13}$ being relative integers. The index $l$ accounts for the "red" $(\mathrm{R})$, the "green" $(\mathrm{G})$, and the "blue" (B) sublattices in Fig.3; $\epsilon_{R}=1, \epsilon_{G}=1 / 2, \epsilon_{B}=1 / 2 ; \delta_{R}=0, \delta_{G}=-1 / 2, \delta_{B}=1 / 2$, while the constants $\xi_{1}, \xi_{2}$ cancel the terms $\propto \mu_{1}, \mu_{2}$ in $\chi_{1}(0), \chi_{2}(0)$, respectively. The spin polarization of $\mathbf{S}_{G}$ is uniform throughout each one of the three sublattices; thus, to the minima of $\mathcal{H}_{\mathbf{B}}$ are associated the states

$$
\begin{aligned}
& |R\rangle=\left(\cos \frac{\theta_{f}}{2}|\Uparrow\rangle+e^{i \frac{\pi}{3}} \sin \frac{\theta_{f}}{2}|\Downarrow\rangle\right), \\
& |G\rangle=\left(\cos \frac{\theta_{f}}{2}|\Uparrow\rangle+e^{-i \frac{\pi}{3}} \sin \frac{\theta_{f}}{2}|\Downarrow\rangle\right), \\
& |B\rangle=\left(\cos \frac{\theta_{f}}{2}|\Uparrow\rangle+e^{i \pi} \sin \frac{\theta_{f}}{2}|\Downarrow\rangle\right),
\end{aligned}
$$

where $\cos \frac{\theta_{f}}{2}=\frac{\sqrt{16+B_{\|}^{2}}-B_{\|}}{\sqrt{16+B_{\|}^{2}-B_{\|} \sqrt{16+B_{\|}^{2}}}}$ and $\sin \frac{\theta_{f}}{2}=\frac{1}{\sqrt{16+B_{\|}^{2}-B_{\|} \sqrt{16+B_{\|}^{2}}}}$. To the first order in $B_{\|}$(i.e. to the first order in $(f-\pi))$ one has:

$$
\theta_{f} \approx \frac{\pi}{2}+\frac{B_{\|}}{4}
$$

From the zero-mode spectrum, one sees that the energy of each field configuration gets a zeromode contribution which is quadratic in $\vec{p}$; inserting the solution of Eq.(49) in the noninteracting Hamiltonian $\mathcal{H}_{\mathrm{LL}}$, one finds the zero-mode contribution to the total energy for each of the three sublattices. Namely, one gets:

\section{- Red-points:}


Zero-mode eigenvalues:

$$
\left(p_{1}, p_{2}\right)_{R}=\sqrt{2 g}\left(-\frac{\mu_{1}}{2 \pi}+n_{12}+1,-\frac{\mu_{2}}{2 \pi}+\frac{1}{\sqrt{3}}\left(2 n_{13}-n_{12}\right)\right),
$$

Zero-mode contribution to the total energy:

$$
E_{R, n_{12}, n_{13}}^{(0)}(\vec{\mu})=\frac{\pi u g}{L}\left[\left(-\frac{\mu_{1}}{2 \pi}+n_{12}+1\right)^{2}+\left(-\frac{\mu_{2}}{2 \pi}+\frac{1}{\sqrt{3}}\left(2 n_{13}-n_{12}\right)\right)^{2}\right] .
$$

\section{- Green-points:}

Zero-mode eigenvalues:

$$
\left(p_{1}, p_{2}\right)_{G}=\sqrt{2 g}\left(-\frac{\mu_{1}}{2 \pi}+n_{12}+\frac{1}{2},-\frac{\mu_{2}}{2 \pi}+\frac{1}{\sqrt{3}}\left(2 n_{13}-n_{12}-\frac{1}{2}\right)\right),
$$

Zero-mode contribution to the total energy:

$$
E_{G, n_{12}, n_{13}}(\vec{\mu})=\frac{\pi u g}{L}\left[\left(-\frac{\mu_{1}}{2 \pi}+n_{12}+\frac{1}{2}\right)^{2}+\left(-\frac{\mu_{2}}{2 \pi}+\frac{1}{\sqrt{3}}\left(2 n_{13}-n_{12}-\frac{1}{2}\right)\right)^{2}\right] .
$$

\section{- Blue-points:}

Zero-mode eigenvalues:

$$
\left(p_{1}, p_{2}\right)_{B}=\sqrt{2 g}\left(-\frac{\mu_{1}}{2 \pi}+n_{12}+\frac{1}{2},-\frac{\mu_{2}}{2 \pi}+\frac{1}{\sqrt{3}}\left(2 n_{13}-n_{12}+\frac{1}{2}\right)\right)
$$

Zero-mode contribution to the total energy:

$$
E_{B, n_{12}, n_{13}}(\vec{\mu})=\frac{\pi u g}{L}\left[\left(-\frac{\mu_{1}}{2 \pi}+n_{12}+\frac{1}{2}\right)^{2}+\left(-\frac{\mu_{2}}{2 \pi}+\frac{1}{\sqrt{3}}\left(2 n_{13}-n_{12}+\frac{1}{2}\right)\right)^{2}\right] .
$$

Through the external phases $\mu_{1}, \mu_{2}$ one adds an effective quadratic potential breaking the degeneracy of the zero-modes. It is quite remarkable that the zero-mode energy spectrum reported in Eqs. $(54,56,58)$ displays, for convenient choices of the applied phases $\mu_{1}, \mu_{2}$, a threefold degeneracy between the three sites lying at the vertices of a single triangle (box in Fig.3). As we shall see in later sections, this feature of a tetrahedral JJN is crucial for its applications to quantum information processing tasks. 


\subsection{Leading boundary perturbation at the SFP}

In order to describe effects of the leading boundary perturbation at the SFP one should rewrite the boundary Hamiltonian in terms of the phase-slip operators representing instanton trajectories connecting nearest neighboring sites on the lattice in Fig.3. For this purpose, it is most convenient to introduce the phase slip field operators $\Theta_{1}(x, t), \Theta_{2}(x, t)$, dual to $\chi_{1}(x, t), \chi_{2}(x, t)$, and, thus, defined by [6]

$$
\frac{1}{u g} \frac{\partial \Theta_{i}(x, t)}{\partial t}=\frac{\partial \chi_{i}(x, t)}{\partial x} \quad, \quad \frac{g}{u} \frac{\partial \chi_{i}(x, t)}{\partial t}=\frac{\partial \Theta_{i}(x, t)}{\partial x} .
$$

In particular, Eqs.(59) imply that $\Theta_{1,2}(x, t)$ obey Neumann boundary conditions at $x=0$ and at $x=L$. As a result, the mode expansion of the instanton fields is given by

$$
\Theta_{j}(x, t)=\sqrt{2 g}\left\{\theta_{0}^{j}+\frac{\pi u t}{L} P_{j}+i \sum_{n \neq 0} \cos \left[\frac{\pi n x}{L}\right] \frac{\tilde{\alpha}_{n}^{j}}{n} e^{-i \frac{\pi}{L} n v t}\right\},
$$

with

$$
\left[P_{i}, \theta_{0}^{j}\right]=-i \delta_{i, j} \quad, \quad\left[\tilde{\alpha}_{n}^{i}, \tilde{\alpha}_{m}^{j}\right]=n \delta_{n+m, 0} \delta_{i, j} \quad .
$$

A quantum tunnelling between two adjacent minima lying on the lattice shown in Fig. 3 involves a quantum jump between different zero mode eigenstates. The quantum phase slip operators corresponding to the allowed quantum jumps, $W_{i}, W_{i}^{\dagger}(i=1,2,3)$, are given by:

$$
\begin{aligned}
& W_{1}^{\dagger}=: \exp \left[\frac{i}{\sqrt{3}} \vec{\rho}_{1} \cdot \vec{\Theta}(0)\right]: \\
& W_{2}^{\dagger}=: \exp \left[\frac{i}{\sqrt{3}} \vec{\rho}_{2} \cdot \vec{\Theta}(0)\right]: \\
& W_{3}^{\dagger}=: \exp \left[\frac{i}{\sqrt{3}} \vec{\rho}_{3} \cdot \vec{\Theta}(0)\right]: .,
\end{aligned}
$$

The vectors $\overrightarrow{\rho_{i}}$ are defined so that $\sqrt{2 g} \frac{\overrightarrow{\rho_{i}}}{\sqrt{3}}$ is the "distance" between nearest neighboring eigenvalues of $\vec{P}$, as determined by the commutation relations

$$
\left[\vec{P}, W_{i}\right]=-\sqrt{\frac{2 g}{3}} \vec{\rho}_{i} W_{i} \quad, \quad\left[\vec{P}, W_{i}^{\dagger}\right]=\sqrt{\frac{2 g}{3}} \vec{\rho}_{i} W_{i}^{\dagger}
$$

which yield

$$
\vec{\rho}_{1}=\left(\begin{array}{c}
\sqrt{3} / 2 \\
1 / 2
\end{array}\right) \quad, \quad \vec{\rho}_{2}=\left(\begin{array}{c}
0 \\
-1
\end{array}\right) \quad, \quad \vec{\rho}_{3}=\left(\begin{array}{c}
-\sqrt{3} / 2 \\
1 / 2
\end{array}\right) .
$$

In Fig. 3 we have represented the quantum jumps between the eigenvales of $\vec{P}$ corresponding to the operators $W_{i}^{\dagger}$; the action of the hermitean conjugate operators $W_{i}$ may be simply represented by reversing the arrows. 
Since a tunnelling event between minima of $\mathcal{H}_{\mathbf{B}}$ involves a rotation in the two dimensional space spanned by the eigenstates of $\mathbf{S}_{G}$, and since the allowed directions of tunnelling from a given minimum depend on the position of this minimum in the Kagome lattice, each quantum phase slip operator has to be multiplied by the spin operator mapping the state $\{|i\rangle\}(i=R, G, B$, onto the other two states. As a result the leading boundary perturbation at the SFP is given by

$$
\tilde{\mathcal{H}}_{\mathbf{B}}=-Y e^{i \gamma}\left\{S_{G R}^{\dagger} W_{1}^{\dagger}+S_{B G}^{\dagger} W_{2}^{\dagger}+S_{B R}^{\dagger} W_{3}^{\dagger}\right\}+\text { h.c. , }
$$

with $S_{i j}$ the operator sending the "j" spin state into the "i" one, while the parameters $Y, \gamma$ are computed in Appendix D. Since the scaling dimension of the operators $\left\{W_{i}, W_{i}^{\dagger}\right\}$ is $\frac{g}{3}$ the running coupling strength for the dual boundary coupling may be defined as $\zeta(L)=y(L) e^{i \gamma}$, with $y(L)=$ $L Y(L)$.

Even if $\zeta$ is, in general, a complex coupling strength, the renormalization group equations may be derived following the standard procedure used in [6] which, starting from Eq.(65), allows to determine the euclidean dual boundary action at the SFP as

$$
\tilde{S}_{\mathbf{B}}=-Y e^{i \gamma} \int_{0}^{\beta} d \tau\left\{S_{G R}^{\dagger}(\tau) W_{1}^{\dagger}+S_{B G}^{\dagger}(\tau) W_{2}^{\dagger}+S_{B R}^{\dagger}(\tau) W_{3}^{\dagger}\right\}+\text { h.c.. }
$$

Here $W_{j}(\tau)=e^{-\frac{i}{\sqrt{3}} \vec{\rho}_{i} \cdot \vec{\Theta}(\tau)}$, with $\Theta(\tau)=\Theta(0, i \tau)$; one may then compute the $\beta$-functions for the boundary coupling strengths $[6,34]$ by resorting to the O.P.E.s

$$
\left[W_{1}^{\dagger} S^{\dagger}\right](\tau)\left[W_{2}^{\dagger} S^{\dagger}\right]\left(\tau^{\prime}\right) \approx_{\tau^{\prime} \rightarrow \tau}\left|\frac{u\left(\tau-\tau^{\prime}\right)}{L}\right|^{-\frac{2 g}{3}}\left[W_{3} S\right](\tau)
$$

plus cyclic permutations.

From Eq.(67), the second-order renormalization group equation for the running coupling $\zeta(L)$ may be written as

$$
\frac{d \zeta(L)}{d \ln \left(\frac{L}{L_{0}}\right)}=\left(1-\frac{g}{3}\right) \zeta(L)-2 e^{-2 i \gamma} \zeta^{2}(L),
$$

where $L_{0}$ is, again, a reference length scale. Eq.(68) is equivalent to the system of real differential equations for the real parameters $y(L), \gamma(L)$ given by

$$
\begin{aligned}
\frac{d y(L)}{d \ln \left(\frac{L}{L_{0}}\right)} & =\left(1-\frac{g}{3}\right) y(L)-2 \cos (3 \gamma) y^{2}(L) \\
y(L) \frac{d \gamma(L)}{d \ln \left(\frac{L}{L_{0}}\right)} & =2 \sin (3 \gamma(L)) y^{2}(L) .
\end{aligned}
$$

From Eqs.(69) one sees that the phase $\gamma$ is renormalized only to the second order in the boundary couplings and that, for $\gamma=k \pi / 3$ ( $k$ integer), there are lines of fixed points in the $y-\gamma$-plane; furthermore, the line $\gamma=\frac{\pi}{3}$ is made of attractive fixed points.

The phase diagram accessible to the tetrahedral JJN may be inferred from the RG equations near the WFP, derived in section 3, and the ones near the SFP, Eqs.(69). As displayed in Fig.4, 


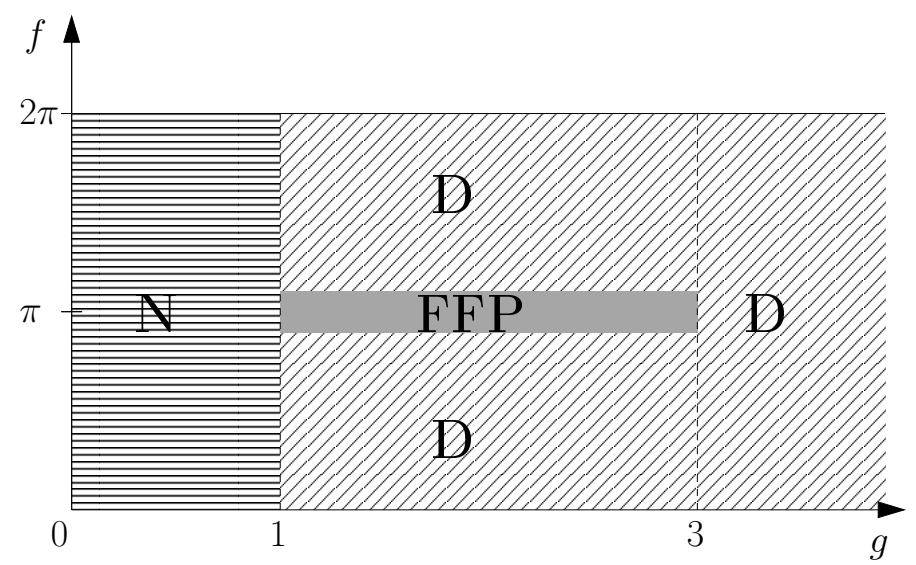

Figure 4: Phase diagram in the strip in the $g, f$-plane corresponding to $0 \leq f \leq 2 \pi$ : the weakly-coupled phase (corresponding to Neumann-(N)- boundary conditions at the inner boundary) is stable for $g<1$ and for any $f$; the strongly-coupled phase (corresponding to Dirichlet-(D)- boundary conditions at the inner boundary) is stable for $g>1$ and for any $f$. For $g<3$ and $-\frac{\pi}{10} \lesssim(f-\pi) \lesssim \frac{\pi}{10}$ a novel phase opens, corresponding to a stable finite coupling fixed point (FFP). The phase diagram for $f \notin[0,2 \pi]$ is obtained by periodical extension of this picture.

for $1<g<3$ and $-\frac{\pi}{10} \lesssim(f-\pi) \lesssim \frac{\pi}{10}^{2}$, neither the WFP, or the SFP, is infrared stable. As a consequence, in this window of values of $f$ and $g$, the infrared behavior of the JJN will be driven by an emerging FFP, whose properties will be analyzed in the next section.

\section{The quantum doublet at the FFP}

In this section we show that the renormalization of the instanton tunnelling strength $Y$, due to the interaction with the plasmon modes of the TLL leads, enforces the emergence of a quantum doublet robust not only against the noise in the external control parameters but also against the decoherence induced by the coupling with the plasmon modes in the leads.

\subsection{The emerging doublet at the FFP}

Since a real device has a finite size $(L)$ the infinite degeneracy induced at the SFP by the eigenvalues of the zero-mode operators, is removed by finite-size effects; i.e., by the zero-mode contributions to the total energy. As a function of the external phases $\vec{\mu}$, the zero-mode energy associated to each eigenvalue $\vec{p}$ is given by

$$
E_{\{R, G, B\}, n_{12}, n_{13}}^{(0)}[\vec{\mu}]=\frac{\pi u}{2 L}\left[\vec{p}\left(n_{12}, n_{13}\right)\right]^{2} .
$$

From Eqs. $(54,56,58)$, one sees that, for a pertinent choice of the phases $\vec{\mu}$, the three zero-mode energies associated to the vertices of a triangle as the one drawn in the box of Fig.3, may be made degenerate. To explicitly show this, let us set $\mu_{1}^{*}=\frac{4 \pi}{3}, \mu_{2}^{*}=0$. For such a choice of the

\footnotetext{
${ }^{2}$ This interval is determined in Sec.6 and accounts for the fact that, for $f \neq \pi$, the renormalization of $B_{\perp}$ may lift the degeneracy between the minima.
} 
external phases, one gets $E_{R, 0,0}^{(0)}\left[\vec{\mu}^{*}\right]=E_{G, 0,0}^{(0)}\left[\vec{\mu}^{*}\right]=E_{B, 0,0}^{(0)}\left[\vec{\mu}^{*}\right]$. Thus, if one restricts himself to the three-dimensional subspace $\mathcal{F}$ of the Hilbert space spanned by the states $|R\rangle,|G\rangle,|B\rangle$, an effective low-energy description of the tetrahedral JJN at the SFP may be provided by the $3 \times 3$ Hamiltonian matrix $\mathcal{H}_{\mathcal{F}}$ given by

$$
\mathcal{H}_{\mathcal{F}}=\frac{\pi g u}{9 L} \mathbf{I}+\left[\begin{array}{ccc}
0 & -Y e^{i \gamma} & -Y e^{-i \gamma} \\
-Y e^{-i \gamma} & 0 & -Y e^{i \gamma} \\
-Y e^{i \gamma} & -Y e^{-i \gamma} & 0
\end{array}\right],
$$

Form (71) one sees that, when $Y \neq 0$, the degeneracy between $|R\rangle,|G\rangle$ and $|B\rangle$ is broken and that the spectrum of the low-lying energy states admits as its groundstate a quantum doublet confined away from a singlet state. To see this, let us consider the two operators acting on $\mathcal{F}$ whose matrix representations are given by

$$
A_{1}=\left[\begin{array}{lll}
0 & 1 & 1 \\
1 & 0 & 1 \\
1 & 1 & 0
\end{array}\right] \quad, \quad A_{2}=\left[\begin{array}{ccc}
1 & 0 & 0 \\
0 & 0 & e^{-i \frac{2}{3} \pi} \\
0 & e^{i \frac{2}{3} \pi} & 0
\end{array}\right] .
$$

While $A_{1}$ corresponds to a cyclic shift of the states $|R\rangle,|G\rangle,|B\rangle, A_{2}$ realizes the mirror inversion about the triangle height passing through the vertex corresponding to the $|R\rangle_{\text {-state. Though, as }}$ expected, when $\gamma=\pi / 3$, both $A_{1}$ and $A_{2}$ commute with $\mathcal{H}_{\mathcal{F}},\left[\mathcal{H}_{\mathcal{F}}, A_{1}\right]=\left[\mathcal{H}_{\mathcal{F}}, A_{2}\right]=0$, they do not commute with each other since $\left[A_{1}, A_{2}\right] \neq 0$. This is enough to ensure that the spectrum of $\mathcal{H}_{\mathcal{F}}$ must contain at least one degenerate eigenvalue. To explicitly check it out, let us consider the eigenvalue equation associated to $\mathcal{H}_{\mathcal{F}}$. It reads

$$
-E^{3}+3 Y^{2} E-2 \cos (3 \gamma) Y^{3}=0
$$

For $\gamma=\pi / 3$ there is a twofold degenerate eigenvalue $E=-Y$, and a non degenerate eigenvalue $E=2 Y$. The corresponding eigenstates are

$$
\begin{aligned}
|-Y\rangle_{1} & =\frac{1}{\sqrt{3}}[|R\rangle+|G\rangle+|B\rangle] \\
|-Y\rangle_{2} & =\frac{1}{\sqrt{3}}\left[|R\rangle+e^{-i \frac{2}{3} \pi}|G\rangle+e^{i \frac{2}{3} \pi}|B\rangle\right] \\
|2 Y\rangle & =\frac{1}{\sqrt{3}}\left[|R\rangle+e^{i \frac{2}{3} \pi}|G\rangle+e^{-i \frac{2}{3} \pi}|B\rangle\right] .
\end{aligned}
$$

To rewrite $\mathcal{H}_{\mathcal{F}}$ using the states given in Eq.(74), one has to rotate it to $\bar{H}_{\mathcal{F}}=P^{\dagger} \mathcal{H}_{\mathcal{F}} P$, with the matrix $P$ given by

$$
P=\frac{1}{\sqrt{3}}\left[\begin{array}{ccc}
1 & 1 & 1 \\
1 & e^{-i \frac{2}{3} \pi} & e^{i \frac{2}{3} \pi} \\
1 & e^{i \frac{2}{3} \pi} & e^{-i \frac{2}{3} \pi}
\end{array}\right]
$$


One obtains

$$
P^{\dagger} \mathcal{H}_{\mathcal{F}} P=\frac{\pi g u}{9 L} \frac{1}{9} \mathbf{I}+\left[\begin{array}{ccc}
-Y & 0 & 0 \\
0 & -Y & 0 \\
0 & 0 & 2 Y
\end{array}\right]
$$

Similarly, by rotating $A_{2}$, one gets

$$
P^{\dagger} A_{2} P=\left[\begin{array}{lll}
0 & 1 & 0 \\
1 & 0 & 0 \\
0 & 0 & 1
\end{array}\right] ;
$$

thus, $A_{2}$ is the operator swapping the degenerate states $|-Y\rangle_{1},|-Y\rangle_{2}$ with each other.

Eq.(76) shows that a quantum doublet- this time robust also against decoherence induced by its coupling with the plasmon fields in the TLL leads- emerges in a tetrahedral JJN operated near the FFP. Indeed, accounting for the fluctuations of the plasmon fields amounts only to substitute $Y$ with the running coupling strength, that is $\sim\left(\frac{L}{L_{0}}\right)^{-\frac{g}{3}}$. Thus, we find that, via renormalization of the boundary coupling strength, the collective plasmon modes renormalize the instanton fugacity so as to make the gap $\Delta$ between the quantum doublet, $|-Y\rangle_{1,2}$, and the first excited singlet, $|2 Y\rangle$, scale like $\Delta(L)=\Delta_{0}\left(\frac{L}{L_{0}}\right)^{-\frac{g}{3}}$. Thus, the interaction with the plasmon modes enforces the gap between the quantum doublet and the first excited state.

\subsection{Manipulation of the quantum doublet at the FFP}

Let us, firstly, assume that the external phases $\vec{\mu}$ are tuned nearby, but not exactly at, the triple degeneracy point, $\mu_{1}^{*}, \mu_{2}^{*}$, that is, $\left|\mu_{1}-\mu_{1}^{*}\right| / \pi \ll 1,\left|\mu_{2}-\mu_{2}^{*}\right| / \pi \ll 1$. The low-energy effective Hamiltonian $\mathcal{H}_{\mathcal{F}}$ in the basis $|R\rangle,|G\rangle,|B\rangle$ is now modified to

$$
\mathcal{H}_{\mathcal{F}}[\vec{\epsilon}]=\left\{\frac{\pi g u}{L}\left[\frac{1}{9}+\frac{\vec{\epsilon}^{2}}{4 \pi^{2}}\right]\right\} \mathbf{I}+\left[\begin{array}{ccc}
-\frac{2}{3} \frac{\pi g u}{L} \frac{\epsilon_{1}}{2 \pi} & -Y e^{i \frac{\pi}{3}} & -Y e^{-i \frac{\pi}{3}} \\
-Y e^{-i \frac{\pi}{3}} & \frac{\pi g u}{L}\left[\frac{1}{3} \frac{\epsilon_{1}}{2 \pi}-\frac{\epsilon_{2}}{\sqrt{3} \pi}\right] & -Y e^{i \frac{\pi}{3}} \\
-Y e^{i \frac{\pi}{3}} & -Y e^{-i \frac{\pi}{3}} & \frac{\pi g u}{L}\left[\frac{1}{3} \frac{\epsilon_{1}}{2 \pi}+\frac{\epsilon_{2}}{\sqrt{3} \pi}\right]
\end{array}\right],
$$

where we have set $\epsilon_{i}=\mu_{i}-\mu_{i}^{*}, i=1,2$. Of course, for $\epsilon_{1}=\epsilon_{2}=0, \mathcal{H}_{\mathcal{F}}[\vec{\epsilon}]$ reduces back to the Hamiltonian in Eq.(71). In order to rewrite $\mathcal{H}_{\mathcal{F}}[\vec{\epsilon}]$ using the states reported in Eq.(74), one has to rotate it to $\bar{H}_{\mathcal{F}}[\vec{\epsilon}]=P^{\dagger} \mathcal{H}_{\mathcal{F}}[\vec{\epsilon}] P$ obtaining

$$
P^{\dagger} \mathcal{H}_{\mathcal{F}}[\vec{\epsilon}] P=\left\{\frac{\pi g u}{L}\left[\frac{1}{9}+\frac{\vec{\epsilon}^{2}}{4 \pi^{2}}\right]\right\} \mathbf{I}+\left[\begin{array}{ccc}
-Y & a-i \frac{b}{\sqrt{3}} & a+i \frac{b}{\sqrt{3}} \\
a+i \frac{b}{\sqrt{3}} & -Y & a-i \frac{b}{\sqrt{3}} \\
a-i \frac{b}{\sqrt{3}} & a+i \frac{b}{\sqrt{3}} & 2 Y
\end{array}\right],
$$

with $a=-\frac{1}{3} \frac{\pi g u}{L} \frac{\epsilon_{1}}{2 \pi}, b=-\frac{\pi g u}{L} \frac{\epsilon_{2}}{\sqrt{3} \pi}$. From Eq.(79), by simply keeping the matrix elements of $P^{\dagger} \mathcal{H}_{\mathcal{F}}[\vec{\epsilon}] P$ involving the low energy twofold degenerate ground state, one has

$$
\mathcal{H}_{\mathrm{Eff}}=\left\{\frac{\pi g u}{L}\left[\frac{1}{9}+\frac{\vec{\epsilon}^{2}}{4 \pi^{2}}\right]-Y\right\} \mathbf{I}_{2}+\left[\begin{array}{cc}
0 & a-i \frac{b}{\sqrt{3}} \\
a+i \frac{b}{\sqrt{3}} & 0
\end{array}\right] .
$$


From Eq.(80), one sees that, apart from the term proportional to the identity matrix, the matrix $\mathcal{H}_{\text {Eff }}$ can be rewritten as $\mathcal{H}_{\text {Eff }}=b_{x} \sigma^{x}+b_{y} \sigma^{y}$, with $b_{x}=a$, and $b_{y}=\frac{b}{\sqrt{3}}$.

To add the $z$-component to the effective magnetic field one needs to break the degeneracy between the $|\Uparrow\rangle$ - and the $|\Downarrow\rangle$-states of $\mathbf{S}_{G}$. This may be realized by turning on a nonzero $B_{\|}$- see Eq.(18)- and amounts to introduce an additional contribution to $\mathcal{H}_{\mathcal{F}}$ given (see Appendix D) by

$$
\delta \mathcal{H}_{\mathcal{F}}=-\frac{B_{\|}}{4} Y\left[\begin{array}{ccc}
0 & z & z^{*} \\
z^{*} & 0 & z \\
z & z^{*} & 0
\end{array}\right]
$$

with $z=\frac{\pi}{3} e^{-i \frac{\pi}{6}}$. When transforming to the states given in Eq.(74), one obtains

$$
P^{\dagger} \delta \mathcal{H}_{\mathcal{F}} P=-\frac{\sqrt{3}}{4} B_{\|} Y\left[\begin{array}{ccc}
1 & 0 & 0 \\
0 & -1 & 0 \\
0 & 0 & 0
\end{array}\right] .
$$

From Eq.(82) one readily gets the low energy effective two states Hamiltonian

$$
\mathcal{H}_{\mathrm{Eff}}[\vec{b}]=\left\{\frac{\pi g u}{L}\left[\frac{1}{9}+\frac{\vec{\epsilon}^{2}}{4 \pi^{2}}\right]-Y\right\} \mathbf{I}_{2}+\vec{b} \cdot \vec{\sigma},
$$

with $b_{x}=a, b_{y}=\frac{b}{\sqrt{3}}$, and $b_{z}=-\frac{\sqrt{3}}{4} B_{\|} Y$. The Hamiltonian $\mathcal{H}_{\mathrm{Eff}}[\vec{b}]$ is then the Hamiltonian for a spin in an external magnetic field, whose components may be manipulated by acting on the external control parameters of the tetrahedral JJN.

For instance, applying a modulation in time to the phases $\vec{\mu}$ and to the flux $f$, one may change the relative sign between the two states according to the procedure outlined in Ref.[26]. Indeed, one may modulate in time $\epsilon_{2}$, so that $\epsilon_{2}(t)=v \sin \left(\omega_{0} t\right)$. This results in an effective $\vec{b}$ field given by $\vec{b}=\left(b_{x}, \tilde{b} \sin \left(\omega_{0} t\right), b_{z}\right)$, with $b_{x}, b_{z}$ constant and $\tilde{b}=\frac{\pi g u}{3 L} v$. The instantaneous eigenvalues of $\mathcal{H}_{\mathrm{Eff}}[\vec{b}(t)]$ are then given by $\pm \Lambda(t)= \pm \sqrt{b_{x}^{2}+b_{z}^{2}+\tilde{b}^{2} \sin ^{2}\left(\omega_{0} t\right)}$, while the corresponding adiabatic eigenstates are given by

$$
\begin{aligned}
|-\Lambda(t)\rangle & =e^{i \xi_{-}(t)}\left\{\cos \left(\frac{\Theta(t)}{2}\right)|-Y\rangle_{1}+\sin \left(\frac{\Theta(t)}{2}\right) e^{-i \Phi(t)}|-Y\rangle_{2}\right\} \\
|\Lambda(t)\rangle & =e^{i \xi_{+}(t)}\left\{-\sin \left(\frac{\Theta(t)}{2}\right)|-Y\rangle_{1}+\cos \left(\frac{\Theta(t)}{2}\right) e^{-i \Phi(t)}|-Y\rangle_{2}\right\}
\end{aligned}
$$

with $\cos (\Theta)=-b_{z} / \Lambda(t)$ and $\Phi(t)=\arg \left(-b_{x}-i \tilde{b} \sin \left(\omega_{0} t\right)\right)$. As usual, the phases $\xi_{ \pm}(t)$ are chosen so as to satisfy the condition

$$
\left\langle\Lambda(t)\left|\frac{d}{d t}\right| \Lambda(t)\right\rangle=\left\langle-\Lambda(t)\left|\frac{d}{d t}\right|-\Lambda(t)\right\rangle=0
$$

Preparing, at $t=0$, the system in the state $-|\Lambda(0)\rangle$, after twice a period $2 T=4 \pi \omega_{0}$, the relative phases between $|-Y\rangle_{1}$ and $|-Y\rangle_{2}$ becomes $\Delta \Phi=-\frac{1}{2} \int_{0}^{4 \pi / \omega_{0}} d t \dot{\Phi}(t)[1-\cos (\Theta(t))]$. Setting $b_{z}=0$ 
and $v=b_{x}$, one finds $\Delta \Phi=\pi$ and, thus, the relative sign between the two states is exchanged. This procedure allows for engineering a NOT port, using the quantum doublet emerging in a tetrahedral JJN at the FFP.

To read the state of the quantum doublet one may look at the pattern of the Josephson currents arising circulating in the JJN when it is biased off the triple-degeneracy point. Indeed, within the restricted subspace $\mathcal{F}$, the current operators are given by

$$
\begin{aligned}
I_{1, \mathcal{F}} & =e^{*} \frac{\partial P^{\dagger} \mathcal{H}_{\mathcal{F}}[\vec{\epsilon}] P}{\partial \epsilon_{1}}=\frac{e^{*} g u}{2 \pi L} \epsilon_{1} \mathbf{I}-\frac{e^{*} g u}{6 L}\left[\begin{array}{ccc}
0 & 1 & 1 \\
1 & 0 & 1 \\
1 & 1 & 0
\end{array}\right] \\
I_{2, \mathcal{F}} & =e^{*} \frac{\partial P^{\dagger} \mathcal{H}_{\mathcal{F}}[\vec{\epsilon}] P}{\partial \epsilon_{2}}=\frac{e^{*} g u}{2 \pi L} \epsilon_{2} \mathbf{I}+i \frac{e^{*} g u}{3 L}\left[\begin{array}{ccc}
0 & 1 & -1 \\
-1 & 0 & 1 \\
1 & -1 & 0
\end{array}\right] .
\end{aligned}
$$

From Eq.(86), one obtains

$$
\begin{aligned}
& { }_{1}\left\langle-Y\left|I_{1, \mathcal{F}}\right|-Y\right\rangle_{1}=\frac{e^{*} g u}{2 \pi L} \epsilon_{1}={ }_{2}\left\langle-Y\left|I_{1, \mathcal{F}}\right|-Y\right\rangle_{2} \\
& { }_{1}\left\langle-Y\left|I_{1, \mathcal{F}}\right|-Y\right\rangle_{2}=-\frac{e^{*} g u}{6 L} \\
& { }_{1}\left\langle-Y\left|I_{2, \mathcal{F}}\right|-Y\right\rangle_{1}=\frac{e^{*} g u}{2 \pi L} \epsilon_{2}={ }_{2}\left\langle-Y\left|I_{2, \mathcal{F}}\right|-Y\right\rangle_{2} \\
& { }_{1}\left\langle-Y\left|I_{2, \mathcal{F}}\right|-Y\right\rangle_{2}=i \frac{e^{*} g u}{3 L}
\end{aligned}
$$

On the generic state of the doublet given by $|\alpha\rangle=\cos \frac{\theta}{2}|-Y\rangle_{1}+e^{i \phi} \sin \frac{\theta}{2}|-Y\rangle_{2}$, the expectation value of the current operators are

$$
\begin{aligned}
& \left\langle\alpha\left|I_{1, \mathcal{F}}\right| \alpha\right\rangle=-\frac{e^{*} g u}{6 L} \frac{1}{2}\left\langle\sigma_{x}\right\rangle_{\alpha}+\frac{e^{*} g u}{2 \pi L} \epsilon_{1} \\
& \left\langle\alpha\left|I_{2, \mathcal{F}}\right| \alpha\right\rangle=-\frac{e^{*} g u}{3 L} \frac{1}{2}\left\langle\sigma_{y}\right\rangle_{\alpha}+\frac{e^{*} g u}{2 \pi L} \epsilon_{2}
\end{aligned}
$$

From Eqs.(B.5) and Eq.(91), it is easy to determine the current pattern identifying each degenerate state $|-Y\rangle_{1},|-Y\rangle_{2}$.

\section{Engineering a tetrahedral JJN operating near the FFP}

Spinless TLL leads may be realized also with classical Josephson junctions - i.e. using junctions for which $E_{J} / E_{c} \geq 1$ - [36], which may be easily and reliably fabricated with well tested technologies [32]. In this realization the Luttinger parameter $g$ is given by $g \sim \sqrt{\pi E_{J} / E_{c}}$ [36] and a tetrahedral JJN operating near the FFP may be fabricated by requiring that $g=2$ and then setting $L_{*} \sim 10^{3}$; this since, for $L \geq L_{*}$ and $1<g<3$, the phase slip operators destabilize the SFP. The requirement $g=2$ may be easily satisfied by using junctions for which $E_{J} / E_{c} \sim 1.3$. 
Choosing $\lambda \sim E_{J} / 3$ (see Eq. (17)) one gets that $L_{*} \sim\left(\frac{E_{J}}{E_{3}}\right)^{\frac{g}{g-1}} L_{0}$ is of order $L_{*} \approx 10^{2} L_{0}$ where $L_{0}$ is the reference length of a chain with parameters $E_{J}, E_{3}$. Setting $L_{0} \sim 10$ yields then $L_{*} \sim 10^{3}$.

Flux noise induced by the shift $\left\{f \rightarrow f+\delta_{i}\right\}$ affects the stability of the quantum doublet since its effects amount to introduce an effective interaction between $\mathbf{S}_{G}$ and a magnetic field $\mathbf{B}$ breaking the degeneracy between the $|R\rangle,|G\rangle$ and $|B\rangle$-states. As shown in subsection 4, these fluctuations are potentially dangerous, as they may induce an effective $\mathbf{B}$-field acting on the spin $\mathbf{S}_{G}$, which breaks the degeneracy between $|R\rangle,|G\rangle$ and $|B\rangle$-states. Since the running coupling strength associated to $B_{\perp}$ scales with $L$ as $b_{\perp}(L)=L B_{\perp}$ one sees that the scale- $L_{\text {Stop }}$ at which " dangerous" instanton trajectories will be suppressed by $B_{\perp}$ may be be defined by the condition $b_{\perp}\left(L_{\text {Stop }}\right) \sim y\left(L_{\text {Stop }}\right)$, from which one gets $L_{\text {Stop }} \sim\left(\frac{Y}{B_{\perp}}\right)^{\frac{3}{g}} L_{0}$.

To provide a rough estimate of $L_{\text {Stop }}$, one may approximate the actual instanton as a doublewell instanton, by fitting the parameters of the double-well potential $V_{\mathrm{DB}}$ so that the minimum and the maximum points (and the values of $V_{\mathrm{DB}}$ at the corresponding points) coincide with the ones obtained from $\mathcal{H}_{\mathbf{B}}$. A standard computation $[23,37]$ allows then to estimate the instanton fugacity as

$$
Y \approx 3 \pi \sqrt{\frac{E_{3} \pi u}{g L}} \exp \left[-4.36 \sqrt{\frac{g L E_{3}}{3 \pi u}}\right] .
$$

Using the same fabrication parameters as before and fixing $\left|\delta_{i}\right| \sim \pi / 20$ (i.e., $B_{\perp} \sim 0.2 E_{J}\left(\delta_{i}\right)^{2}$ ), one gets that $L_{\text {Stop }} \sim 8 \times 10^{3}$. As a result, one may infer that noise in the external flux $f$ (described in our approach by $B_{\perp} \neq 0$ and by a finite $L_{\text {Stop }}$ ) does not affect the quantum doublet provided that $L_{\text {Stop }}>L_{*}$.

\section{Concluding Remarks}

In this paper we analyzed the phases accessible to a tetrahedral JJN made by coupling a tetrahedral qubit $[26,27]$ to three JJ chains acting as TLL leads.

We showed that, in a pertinent range of the fabrication and control parameters, a robust attractive FFP emerges due to the geometry of the tetrahedral JJN . In our approach the central region made by the tetrahedral qubit- is treated as a quantum impurity of this low dimensional network. As a result, the emergence of a FFP is a non perturbative phenomenon arising from the strong coupling of the impurity with the TLL superconducting leads.

We argued that the new stable FFP is associated with the emergence of a doubly degenerate ground state, which may be regarded as a quantum doublet described by a spin $1 / 2$ degree of freedom, coupled to the plasmon modes of the superconducting TLL leads via the boundary interaction. We showed that this quantum doublet is robust not only against the noise in the external control parameters (magnetic flux, gate voltage) but also against the decoherence induced by the coupling of the tetrahedral qubit with the superconducting leads. For this purpose, we showed that, as the network size increases, the instanton operators, arising from the interaction of the central region with the plasmon modes of the leads, contribute to enforce the energy gap between the twofold degenerate ground state and the first excited state. 
We also pointed out how one may device protocols allowing to read and manipulate the state of the quantum doublet emerging at the FFP; we saw that this may be achieved by connecting the tetrahedral JJN to three bulk superconductors at fixed phases $\left\{\varphi_{j}\right\}(j=1,2,3$ - see Fig.2). Indeed, we showed that, acting on the $\left\{\varphi_{j}\right\}$, induces an "effective magnetic field", which couples to the emerging two-level quantum system, providing a tool to prepare the two-level quantum system in a given state.

Finally, it is worth to point out that superconducting devices such as the tetrahedral Josephson junction network analyzed in this paper may be used to simulate physical behaviors realizable in Kondo systems. Indeed, our RG analysis showed that, for $g=1$ and $G_{z}(l=0)=0$, the tetrahedral JJN may be used to simulate a Kondo spin pertinently coupled to band electrons.

\section{Acknowledgements}

We thank Ian Affleck and Alioscia Hamma for enlightening discussions at various stages of our research. We benefited from conversations with Maria Cristina Diamantini, Gianluca Grignani, Mario Rasetti and Andrea Trombettoni. This work has been partly supported by INFN.

\section{Appendix A. The central region energy eigenstates}

In this appendix we report the full spectrum of the Hamiltonian given in Eq.(1), for a generic value of the applied flux $f$. In particular, we will single out the twofold degenerate ground state whose levels have been used in section 2.2 , to define the effective spin-1/2 operator $\mathbf{S}_{G}$. The eigenstates, together with the corresponding energy eigenvalues, are given by ${ }^{3}$ :

$\boldsymbol{m}=\mathbf{2}$ : a fully polarized spin-2 state:

$$
|2\rangle=|\uparrow \uparrow \uparrow \uparrow\rangle, \quad\left(\varepsilon_{2}=-2 H\right)
$$

$\boldsymbol{m}=\mathbf{1}$ : four states given by

$$
\begin{aligned}
|1,1\rangle & =\frac{1}{\sqrt{2 t(t-\cos f)}}[(-\cos f+t)|\downarrow \uparrow \uparrow \uparrow\rangle+|\uparrow \downarrow \uparrow \uparrow\rangle+|\uparrow \uparrow \downarrow \uparrow\rangle+|\uparrow \uparrow \uparrow \downarrow\rangle] \\
\varepsilon_{1,1}(f) & =-H+\frac{E_{J}}{2}\left(-\cos f-\sqrt{3+\cos ^{2} f}\right) \\
|1,2\rangle & =\frac{1}{\sqrt{2 t(t+\cos f)}}[(-\cos f-t)|\downarrow \uparrow \uparrow \uparrow\rangle+|\uparrow \downarrow \uparrow \uparrow\rangle+|\uparrow \uparrow \downarrow \uparrow\rangle+|\uparrow \uparrow \uparrow \downarrow\rangle] \\
\varepsilon_{1,2}(f) & =-H+\frac{E_{J}}{2}\left(-\cos f+\sqrt{3+\cos ^{2} f}\right) \\
|1,3\rangle & =\frac{1}{\sqrt{3}}\left[|\uparrow \downarrow \uparrow \uparrow\rangle+e^{i \frac{2 \pi}{3}}|\uparrow \uparrow \downarrow \uparrow\rangle+e^{-i \frac{2 \pi}{3}}|\uparrow \uparrow \uparrow \downarrow\rangle\right]
\end{aligned}
$$

\footnotetext{
${ }^{3}$ notice that the spin labels correspond to sites $0,1,2,3$, respectively; we shall set $s=\sqrt{1+3 \sin ^{2} f}$ and $t=$ $\sqrt{3+\cos ^{2} f}$ henceforth; moreover we shall label the energy eigenstates by means of two quantum numbers: the former ones refer to the total spin momentum of the states, the latter ones to the $z$-component of the total spin
} 


$$
\begin{aligned}
\varepsilon_{1,3}(f) & =-H+\frac{E_{J}}{2}(\cos f-\sqrt{3} \sin f) \\
|1,4\rangle & =\frac{1}{\sqrt{3}}\left[|\uparrow \downarrow \uparrow \uparrow\rangle+e^{-i \frac{2 \pi}{3}}|\uparrow \uparrow \downarrow \uparrow\rangle+e^{i \frac{2 \pi}{3}}|\uparrow \uparrow \uparrow \downarrow\rangle\right] \\
\varepsilon_{1,4}(f) & =-H+\frac{E_{J}}{2}(\cos f+\sqrt{3} \sin f)
\end{aligned}
$$

$\boldsymbol{m}=\mathbf{0}$ : six states given by

$$
\begin{aligned}
& |0,1\rangle=\frac{1}{\sqrt{6 s(s-\sqrt{3} \sin f)}}\left[\left(|\downarrow \downarrow \uparrow \uparrow\rangle+e^{i \frac{2 \pi}{3}}|\downarrow \uparrow \downarrow \uparrow\rangle+e^{-i \frac{2 \pi}{3}}|\downarrow \uparrow \uparrow \downarrow\rangle\right)\right. \\
& \left.+(\sqrt{3} \sin f-s)\left(|\uparrow \uparrow \downarrow \downarrow\rangle+e^{i \frac{2 \pi}{3}}|\uparrow \downarrow \uparrow \downarrow\rangle+e^{-i \frac{2 \pi}{3}}|\uparrow \downarrow \downarrow \uparrow\rangle\right)\right] \\
& \varepsilon_{0,1}(f)=\frac{E_{J}}{2}\left(\cos f-\sqrt{1+3 \sin ^{2} f}\right) \\
& |0,2\rangle=\frac{1}{\sqrt{6 s(s-\sqrt{3} \sin f)}}\left[(\sqrt{3} \sin f-s)\left(|\downarrow \downarrow \uparrow \uparrow\rangle+e^{-i \frac{2 \pi}{3}}|\downarrow \uparrow \downarrow \uparrow\rangle+e^{i \frac{2 \pi}{3}}|\downarrow \uparrow \uparrow \downarrow\rangle\right)\right. \\
& \left.+\left(|\uparrow \uparrow \downarrow \downarrow\rangle+e^{-i \frac{2 \pi}{3}}|\uparrow \downarrow \uparrow \downarrow\rangle+e^{i \frac{2 \pi}{3}}|\uparrow \downarrow \downarrow \uparrow\rangle\right)\right] \\
& \varepsilon_{0,2}(f)=\varepsilon_{0,1}(f) \\
& |0,3\rangle=\frac{1}{\sqrt{6 s(s+\sqrt{3} \sin f)}}\left[\left(|\downarrow \downarrow \uparrow \uparrow\rangle+e^{i \frac{2 \pi}{3}}|\downarrow \uparrow \downarrow \uparrow\rangle+e^{-i \frac{2 \pi}{3}}|\downarrow \uparrow \uparrow \downarrow\rangle\right)\right. \\
& \left.+(\sqrt{3} \sin f+s)\left(|\uparrow \uparrow \downarrow \downarrow\rangle+e^{i \frac{2 \pi}{3}}|\uparrow \downarrow \uparrow \downarrow\rangle+e^{-i \frac{2 \pi}{3}}|\uparrow \downarrow \downarrow \uparrow\rangle\right)\right] \\
& \varepsilon_{0,3}(f)=\frac{E_{J}}{2}\left(\cos f+\sqrt{1+3 \sin ^{2} f}\right) \\
& |0,4\rangle=\frac{1}{\sqrt{6 s(s+\sqrt{3} \sin f)}}\left[(\sqrt{3} \sin f+s)\left(|\downarrow \downarrow \uparrow \uparrow\rangle+e^{-i \frac{2 \pi}{3}}|\downarrow \uparrow \downarrow \uparrow\rangle+e^{i \frac{2 \pi}{3}}|\downarrow \uparrow \uparrow \downarrow\rangle\right)\right. \\
& \left.+\left(|\uparrow \uparrow \downarrow \downarrow\rangle+e^{-i \frac{2 \pi}{3}}|\uparrow \downarrow \uparrow \downarrow\rangle+e^{i \frac{2 \pi}{3}}|\uparrow \downarrow \downarrow \uparrow\rangle\right)\right] \\
& \varepsilon_{0,4}(f)=\varepsilon_{0,3}(f) \\
& |0,5\rangle=\frac{1}{\sqrt{6}}[(|\downarrow \downarrow \uparrow \uparrow\rangle+|\downarrow \uparrow \downarrow \uparrow\rangle+|\downarrow \uparrow \uparrow \downarrow\rangle)-(|\uparrow \uparrow \downarrow \downarrow\rangle+|\uparrow \downarrow \uparrow \downarrow\rangle+|\uparrow \downarrow \downarrow \uparrow\rangle)] \\
& \varepsilon_{0,5}(f)=-E_{J}(\cos f-1) \\
& 1|0,6\rangle=\frac{1}{\sqrt{6}}[(|\downarrow \downarrow \uparrow \uparrow\rangle+|\downarrow \uparrow \downarrow \uparrow\rangle+|\downarrow \uparrow \uparrow \downarrow\rangle)+(|\uparrow \uparrow \downarrow \downarrow\rangle+|\uparrow \downarrow \uparrow \downarrow\rangle+|\uparrow \downarrow \downarrow \uparrow\rangle)] \\
& \varepsilon_{0,6}(f)=-E_{J}(\cos f+1) \text {. }
\end{aligned}
$$


$\boldsymbol{m}=\mathbf{- 1}$ : four states given by

$$
\begin{aligned}
& |-1,1\rangle=\frac{1}{\sqrt{2 t(t-\cos f)}}[(-\cos f+t)|\uparrow \downarrow \downarrow \downarrow\rangle+|\downarrow \uparrow \downarrow \downarrow\rangle+|\downarrow \downarrow \uparrow \downarrow\rangle+|\downarrow \downarrow \downarrow \uparrow\rangle] \\
& \varepsilon_{-1,1}(f)=H+\frac{E_{J}}{2}\left(-\cos f-\sqrt{3+\cos ^{2} f}\right) \\
& |-1,2\rangle=\frac{1}{\sqrt{2 t(t+\cos f)}}[(-\cos f-t)|\uparrow \downarrow \downarrow \downarrow\rangle+|\downarrow \uparrow \downarrow \downarrow\rangle+|\downarrow \downarrow \uparrow \downarrow\rangle+|\downarrow \downarrow \downarrow \uparrow\rangle] \\
& \varepsilon_{-1,2}(f)=H+\frac{E_{J}}{2}\left(-\cos f+\sqrt{3+\cos ^{2} f}\right) \\
& |-1,3\rangle=\frac{1}{\sqrt{3}}\left[|\downarrow \uparrow \downarrow \downarrow\rangle+e^{-i \frac{2 \pi}{3}}|\downarrow \downarrow \uparrow \downarrow\rangle+e^{i \frac{2 \pi}{3}}|\downarrow \downarrow \downarrow \uparrow\rangle\right] \\
& \varepsilon_{-1,3}(f)=H+\frac{E_{J}}{2}(\cos f-\sqrt{3} \sin f) \\
& |-1,4\rangle=\frac{1}{\sqrt{3}}\left[|\downarrow \uparrow \downarrow \downarrow\rangle+e^{i \frac{2 \pi}{3}}|\downarrow \downarrow \uparrow \downarrow\rangle+e^{-i \frac{2 \pi}{3}}|\downarrow \downarrow \downarrow \uparrow\rangle\right] \\
& \varepsilon_{-1,4}(f)=H+\frac{E_{J}}{2}(\cos f+\sqrt{3} \sin f) .
\end{aligned}
$$

$\boldsymbol{m}=\mathbf{- 2}$ : this is again a fully polarized spin state given by

$$
|-2\rangle=|\downarrow \downarrow \downarrow \downarrow\rangle, \quad\left(\varepsilon_{-2}=2 H\right)
$$

From the knowledge of these states the effective Hamiltonian for the central region $\mathbf{T}$ given in section 2.1 may be easily derived.

\section{Appendix B. DC-Josephson current pattern at weak couplings}

To induce a DC-Josephson current pattern across the JJN, one has to apply static phase differences to the end point of the network. Thus, the currents may be easily computed within imaginary time path integral formalism discussed in section 3.1. Indeed, if at the endpoint of branch $i$ a static phase $\varphi_{i}$ is applied, the currents $I_{1}, I_{2}, I_{3}$ flowing across the three branches of the JJN may be computed by taking the logarithmic derivatives of the partition function $\mathcal{Z}$ in Eq.(23) with respect to $\mu_{1}, \mu_{2}$ [24], and are given by

$$
\begin{aligned}
& I_{1}=\frac{-e^{*}}{\beta}\left\{\sqrt{2} \frac{\partial \ln \mathcal{Z}}{\partial \mu_{1}}-\sqrt{6} \frac{\partial \ln \mathcal{Z}}{\partial \mu_{2}}\right\} \\
& I_{2}=\frac{-e^{*}}{\beta}\left\{-\sqrt{2} \frac{\partial \ln \mathcal{Z}}{\partial \mu_{1}}-\sqrt{6} \frac{\partial \ln \mathcal{Z}}{\partial \mu_{2}}\right\} \\
& I_{3}=\frac{-e^{*}}{\beta} \sqrt{6} \frac{\partial \ln \mathcal{Z}}{\partial \mu_{2}} .
\end{aligned}
$$


To compute $\mathcal{Z}$, one has to sum over the oscillating modes of the fields $\chi_{j}$, by pertinently taking into account boundary conditions at both boundaries. At the inner boundary $(x=0)$, these are determined by energy conservation and are given by

$$
\begin{aligned}
\frac{u g}{2 \pi} \frac{\partial \vec{\chi}(0, \tau)}{\partial x}= & 2 \bar{E}_{1} \sum_{j} \vec{\alpha}_{j} \sin \left[\vec{\alpha}_{j} \cdot \vec{\chi}(0, \tau)\right]+4 \bar{E}_{z} \sum_{j} \vec{\alpha}_{j} \mathbf{S}_{G}^{z} \sin \left[\vec{\alpha}_{j} \cdot \vec{\chi}(0, \tau)+\frac{\pi}{2}\right]+ \\
& +4 \bar{E}_{3} \sum_{j} \vec{\alpha}_{j} \mathbf{S}_{G}^{x} \cos \left[\frac{2 \pi}{3}(j-2)\right] \sin \left[\vec{\alpha}_{j} \cdot \vec{\chi}(0, \tau)\right]+ \\
& +4 \bar{E}_{3} \sum_{j} \vec{\alpha}_{j} \mathbf{S}_{G}^{y} \sin \left[\frac{2 \pi}{3}(j-2)\right] \sin \left[\vec{\alpha}_{j} \cdot \vec{\chi}(0, \tau)\right]
\end{aligned}
$$

where, in order to account for normal ordering of boundary interaction operators, the boundary interaction strengths have been redefined as $\bar{E}_{\ell}=\left(\frac{a}{L}\right)^{\frac{1}{g}} E_{\ell},(\ell=1, z, 3)[6,18]$. From Eq.(B.2), one easily sees that, at the WFP, energy conservation requires Neumann boundary conditions, for the plasmon fields at $x=0$ (i.e. $\frac{\partial \chi_{1}(0, \tau)}{\partial x}=\frac{\partial \chi_{2}(0, \tau)}{\partial x}=0$ ). As evidenced in section 4.1, at the outer boundary $(x=L) \chi_{1}, \chi_{2}$ obey Dirichlet boundary conditions: $\chi_{1}(L, \tau)=\mu_{1}, \chi_{2}(L, \tau)=\mu_{2}$. As a result, the mode expansion of the fields $\chi_{j}(x, \tau)$ is

$$
\begin{aligned}
\chi_{j}(x, t) & =\mu_{j}+\sqrt{\frac{2}{g}} \sum_{n \in \mathbf{Z}} \cos \left[\frac{\pi}{L}\left(n+\frac{1}{2}\right) x\right] \frac{\alpha_{j}(n)}{n+\frac{1}{2}} e^{-\frac{\pi}{L}\left(n+\frac{1}{2}\right) u \tau} \\
& \equiv \mu_{j}+\phi_{j}(x, \tau),
\end{aligned}
$$

where the oscillator modes $\alpha_{i}(n)$ satisfy the algebra

$$
\left[\alpha_{i}(n), \alpha_{j}(m)\right]=\delta_{i, j} \delta_{n+m-1,0}\left(n+\frac{1}{2}\right) .
$$

For our purposes it is convenient to define "spin-1/2 current operators", that is, operators acting on the two-dimensional Hilbert space of $\mathbf{S}_{G}$ and giving the correct value of the current, when $\mathbf{S}_{G}$ is averaged over, as well. It is straightforward to see that these operators are given by

$$
\begin{aligned}
& I_{1}=-\frac{e^{*}}{\beta}\left\{\sqrt{2} \frac{\partial \ln \mathcal{Z}_{S}}{\partial \mu_{1}}-\sqrt{6} \frac{\partial \ln \mathcal{Z}_{S}}{\partial \mu_{2}}\right\} \\
& I_{2}=-\frac{e^{*}}{\beta}\left\{-\sqrt{2} \frac{\partial \ln \mathcal{Z}_{S}}{\partial \mu_{1}}-\sqrt{6} \frac{\partial \ln \mathcal{Z}_{S}}{\partial \mu_{2}}\right\} \\
& I_{3}=-\frac{e^{*}}{\beta} \sqrt{6} \frac{\partial \ln \mathcal{Z}_{S}}{\partial \mu_{2}},
\end{aligned}
$$

with

$$
\mathcal{Z}_{S}=\int \prod_{i=1,2} \mathcal{D} \chi_{j} e^{-S_{\text {Lead }}-S_{\mathbf{B}}^{(I)}-S_{S}}
$$


Relying over the weak coupling assumption, one may sum over the plasmon modes $\chi_{j}$ within a mean-field like approach. In particular, because of the mode expansion in Eq.(B.3), one gets

$$
\left\langle\cos \left[\vec{\alpha}_{j} \cdot \vec{\chi}(\tau)\right]\right\rangle=\cos \left[\vec{\alpha}_{j} \cdot \vec{\mu}\right] \quad, \quad\left\langle\sin \left[\vec{\alpha}_{j} \cdot \vec{\chi}(\tau)\right]\right\rangle=\sin \left[\vec{\alpha}_{j} \cdot \vec{\mu}\right] \quad .
$$

Thus, one readily sees that, resorting to the mean-field approximation amounts to trade $\vec{\chi}(\tau)$ for the applied phase differences $\vec{\mu}$. As a result, one obtains

$$
\begin{aligned}
\frac{-e^{*}}{\beta} \frac{\partial \ln \mathcal{Z}_{S}}{\partial \vec{\mu}} & =2 e^{*} \bar{E}_{1} \sum_{j} \vec{\alpha}_{j} \sin \left[\vec{\alpha}_{j} \cdot \vec{\mu}\right]+4 e^{*} \bar{E}_{z} \sum_{j} \vec{\alpha}_{j} \mathbf{S}_{G}^{z} \cos \left[\vec{\alpha}_{j} \cdot \vec{\mu}\right]+ \\
& +4 e^{*} \bar{E}_{3} \sum_{j} \vec{\alpha}_{j} \mathbf{S}_{G}^{x} \cos \left(\frac{2 \pi}{3}(j-2)\right) \sin \left[\vec{\alpha}_{j} \cdot \vec{\mu}\right]+ \\
& +4 e^{*} \bar{E}_{3} \sum_{j} \vec{\alpha}_{j} \mathbf{S}_{G}^{y} \sin \left(\frac{2 \pi}{3}(j-2)\right) \sin \left[\vec{\alpha}_{j} \cdot \vec{\mu}\right]
\end{aligned}
$$

There are two possible ways of interpreting Eq.(B.8): on one hand, one may regard the applied phases (and the induced currents) as a probe of the two-level state (which may be set by acting upon it with the external fields $B_{\|}, B_{\perp}$ ). For instance, assuming that the system lies within either one of the eigenstates of $\mathbf{S}_{G}^{z},|\Uparrow\rangle,|\Downarrow\rangle$, and computing the average values of the spin operators as outlined in Appendix C, from Eq.(B.8) one gets

$$
\left\langle\frac{\partial \ln \mathcal{Z}_{S}}{\partial \vec{\mu}}\right\rangle_{S}=2 \sum_{j} \vec{\alpha}_{j}\left\{\bar{E}_{1} \sin \left[\vec{\alpha}_{j} \cdot \vec{\mu}\right] \pm \bar{E}_{z} \cos \left[\vec{\alpha}_{j} \cdot \vec{\mu}\right]\right\}
$$

where the average is computed over the spin coordinates. The corresponding current pattern may be derived from Eq.(B.5) and from Eq.(B.9): clearly, it discriminates between the two states $\mid \Uparrow$ \rangle,$|\Downarrow\rangle$. The same procedure may be applied to probing a generic state of $\mathbf{S}_{G}$, obviously as long as the modification in the state induced by the application of the phases $\vec{\mu}$ is negligible. On the other hand, when no other fields are applied to $\mathbf{S}_{G}$ (that is, when $B_{\|}=B_{\perp}=0$ ), the phases (and, of course, the currents) themselves may be regarded as defining an effective applied field $\mathbf{B}$, whose components are given by

$$
\begin{aligned}
\mathbf{B}^{x} & =-4 \bar{E}_{3} \sum_{j} \cos \left[\frac{2 \pi}{3}(j-2)\right] \cos \left[\vec{\alpha}_{j} \cdot \vec{\mu}\right] \\
\mathbf{B}^{y} & =-4 \bar{E}_{3} \sum_{j} \sin \left[\frac{2 \pi}{3}(j-2)\right] \cos \left[\vec{\alpha}_{j} \cdot \vec{\mu}\right] \\
\mathbf{B}^{z} & =+4 \bar{E}_{z} \sum_{j} \sin \left[\vec{\alpha}_{j} \cdot \vec{\mu}\right] .
\end{aligned}
$$

In this case the phases may be used to drive the state of the two-level system, just as a local magnetic field applied to a true spin-1/2 variable. 


\section{Appendix C. The imaginary time action for a quantum spin-1/2 variable}

In this appendix we shall review the derivation of the imaginary time path integral formalism for a quantum spin-1/2 variable $\mathbf{S}_{G}$ since it has been used to determine the instanton phases at the SFP.

The starting point is the Euclidean action $\mathcal{Z}_{\text {Spin }}$ for a spin- $1 / 2$ degree of freedom in an external magnetic field $\overrightarrow{\mathcal{B}}$, whose dynamics is described by the Hamiltonian

$$
\mathcal{H}_{\text {Spin }}=-\overrightarrow{\mathcal{B}} \cdot \mathbf{S}_{G}
$$

A crucial step is the decomposition of the identity $\mathbf{I}$ in the basis of the coherent states $|\Phi, \Theta\rangle$ as

$$
|\Phi, \Theta\rangle=e^{i \Phi} \cos \left(\frac{\Theta}{2}\right)|\Uparrow\rangle+\sin \left(\frac{\Theta}{2}\right)|\Downarrow\rangle,
$$

The average values of the components of the spin- $1 / 2$ variable on the state $|\Phi, \Theta\rangle$, are then given by

$$
\begin{gathered}
\left\langle\Phi, \Theta\left|\mathbf{S}_{G}^{x}\right| \Phi, \Theta\right\rangle=\frac{1}{2} \cos (\Phi) \sin (\Theta) \quad, \quad\left\langle\Phi, \Theta\left|\mathbf{S}_{G}^{y}\right| \Phi, \Theta\right\rangle=\frac{1}{2} \sin (\Phi) \sin (\Theta) \\
\left\langle\Phi, \Theta\left|\mathbf{S}_{G}^{z}\right| \Phi, \Theta\right\rangle=\frac{1}{2} \cos (\Theta) .
\end{gathered}
$$

In particular, the decomposition of the identity is given by

$$
\mathbf{I}=\int d \Omega|\Phi, \Theta\rangle\langle\Phi, \Theta|
$$

with

$$
\int d \Omega \ldots=\frac{1}{4 \pi} \int_{0}^{2 \pi} d \Phi \int_{0}^{\pi} \sin (\Theta) d \Theta \ldots .
$$

Though coherent states form a complete set, they are not orthogonal to each other; thus, in the imaginary time path integral formulation [38], one has to take into account the overlap amplitude between two coherent states

$$
\left\langle\Phi_{1}, \Theta_{1} \mid \Phi_{2}, \Theta_{2}\right\rangle=e^{-i\left[\Phi_{1}-\Phi_{2}\right]} \cos \left(\frac{\Theta_{1}}{2}\right) \cos \left(\frac{\Theta_{2}}{2}\right)+\sin \left(\frac{\Theta_{1}}{2}\right) \sin \left(\frac{\Theta_{2}}{2}\right) .
$$

Taking into account Eq.(C.6), one gets that the amplitude for $\mathbf{S}_{G}$ to tunnel from the state $\left|\Phi_{0}, \Theta_{0}\right\rangle$ to the state $\left|\Phi_{1}, \Theta_{1}\right\rangle$ in an (imaginary) time $\tau$ is given by

$$
\begin{gathered}
\left\langle\Phi_{1}, \Theta_{1}\left|e^{-\tau H\left[\mathbf{S}_{G}\right]}\right| \Phi_{0}, \Theta_{0}\right\rangle=\int \mathcal{D} \Omega(\tau) e^{\left[-\frac{i}{2} \int_{0}^{\beta} d \tau \dot{\Phi}(\tau)\left(1-\cos (\Theta(\tau))+\int_{0}^{\beta} d \tau \overrightarrow{\mathcal{B}} \cdot \mathbf{S}_{G}(\tau)\right]\right.} \\
\equiv \int \mathcal{D} \Omega(\tau) \exp \left[-S_{E}[\Phi, \Theta]\right]
\end{gathered}
$$


where $H[\mathbf{S}]$ is the spin Hamiltonian, while we have defined the polar angles as in Eqs.(C.3), and the path integral has to be computed over imaginary time trajectories satisfying the boundary conditions $\Theta(0)=\Theta_{0}, \Theta(\tau)=\Theta_{1}$, and $\Phi(0)=\Phi_{0}, \Phi(\tau)=\Phi_{1}$.

The one- and two-spin imaginary time correlation functions we used in section 3 may be then derived by means of a saddle-point approximation. As an example, let us consider the case in which the applied field $B$ is uniform and directed along the $z$-axis, corresponding to Eq.(C.7) for the imaginary time amplitude. The saddle-point equations for the functions $\Phi(\tau), \Theta(\tau)$ are given by:

$$
\begin{aligned}
& 0=\frac{\delta S_{E}[\Phi, \Theta]}{\delta \Theta(\tau)}=\frac{1}{2} \sin (\Theta(\tau))[i \dot{\Phi}(\tau)+B] \\
& 0=\frac{\delta S_{E}[\Phi, \Theta]}{\delta \Phi(\tau)}=\frac{i}{2} \dot{\Theta}(\tau) \sin (\Theta(\tau)) .
\end{aligned}
$$

Eqs.(C.8) imply

$$
\Theta(\tau)=\text { constant }=\Theta_{0}=\Theta_{1} \quad, \quad \Phi(\tau)=i B \tau+\Phi_{0} .
$$

From Eqs.(C.7,C.9) one finds out that, in the saddle-point approximation,

$$
\left\langle\Phi_{1}, \Theta_{1}\left|e^{-\tau H\left[\mathbf{S}_{G}\right]}\right| \Phi_{0}, \Theta_{0}\right\rangle=\delta\left(\Theta_{0}-\Theta_{1}\right) \delta\left(\Phi_{f}-\Phi_{i}-i B \tau\right) e^{\frac{B \tau}{2}} .
$$

From Eq.(C.10) and from the identity

$$
\left\langle\sigma\left|e^{-\tau H}\right| \sigma^{\prime}\right\rangle=\frac{1}{(4 \pi)^{2}} \int_{0}^{2 \pi} d \Phi_{0} d \Phi_{1} \int_{0}^{\pi} d \Theta_{0} d \Theta_{1} \sin \left(\Theta_{0}\right) \sin \left(\Theta_{1}\right)\left\langle\Phi_{1}, \Theta_{1}\left|e^{-\tau H\left[\mathbf{S}_{G}\right]}\right| \Phi_{0}, \Theta_{0}\right\rangle,
$$

with $\mathbf{S}_{G}^{z}|\sigma\rangle=\sigma|\sigma\rangle$, one finds out (assuming $B>0$ ) that

$$
\left\langle\sigma\left|e^{-\tau H}\right| \sigma^{\prime}\right\rangle=e^{-\frac{\sigma B}{2}}
$$

All the other imaginary time average values listed in Eqs.(32,34a) may be derived following a similar approach. For instance, to compute the average value of a component of $\mathbf{S}_{G}$, one may use

$$
\left\langle\mathbf{S}_{G}^{a}(\tau)\right\rangle=\sum_{\sigma}\left\langle\sigma\left|e^{\tau H} \mathbf{S}_{G}^{a} e^{-\tau H}\right| \sigma\right\rangle e^{-\frac{\sigma B}{2}} / \sum_{\sigma} e^{-\frac{\sigma B}{2}}
$$

and

$$
\begin{gathered}
\left\langle\sigma\left|e^{\tau H} \mathbf{S}_{G}^{a} e^{-\tau H}\right| \sigma\right\rangle=\frac{1}{(4 \pi)^{2}} \int_{0}^{2 \pi} d \Phi_{a} d \Phi_{b} \int_{0}^{\pi} d \Theta_{a} d \Theta_{b} \sin \left(\Theta_{a}\right) \sin \left(\Theta_{b}\right) e^{-\frac{\sigma B \tau}{2}}\left\langle\sigma \mid \Phi_{a}, \Theta_{a}\right\rangle \times \\
\left\langle\Phi_{a}, \Theta_{a}\left|\mathbf{S}_{G}^{a}\right| \Phi_{b}, \Theta_{b}\right\rangle\left\langle\Phi_{b}, \Theta_{b} \mid \sigma\right\rangle e^{\frac{\sigma B \tau}{2}}
\end{gathered}
$$

to get the results given in Eqs.(32,34a). When $B_{\|} \neq 0$ one may use a similar analysis, provided one chooses the $z$ axis directed along the direction of $\vec{B}$ and rotates the components of $\mathbf{S}_{G}$ accordingly. 


\section{Appendix D. Derivation of the modulus and phase for instanton trajectories}

In this appendix, we derive the modulus $Y$ and the phase $\gamma$ of the instanton tunnelling amplitudes.

First of all we recall that the instanton trajectory may be regarded as the imaginary time evolution, $\vec{P}=\vec{P}(\tau)$, of the zero mode contribution to Eq.(refeq:mode-exp-strong); it describes a tunnelling event between nearest neighboring sites on the lattice of the minima. The "bulk" Euclidean action for the field $\vec{\chi}$

$$
S^{(0)}=\frac{g}{4 \pi} \int_{0}^{\beta} d \tau \int_{0}^{L} d x\left[\frac{1}{u}\left(\frac{\partial \vec{\chi}}{\partial \tau}\right)^{2}+u\left(\frac{\partial \vec{\chi}}{\partial x}\right)^{2}\right],
$$

yields

$$
S^{(0)}=\frac{1}{2} \int_{0}^{\beta} d \tau\left[M\left(\frac{d \vec{\chi}(\tau)}{d \tau}\right)^{2}+M \omega^{2}(\vec{\chi}(\tau)-\vec{\xi})^{2}\right]+\ldots \equiv S^{(0)}[\vec{\chi}(\tau)]+\ldots
$$

with $\vec{\chi}(\tau)=\vec{\chi}(0, \tau), M=L g /(6 \pi u)$, and $M \omega^{2}=u g /(2 \pi L)$. The ellipses in Eqs.(D.1,D.2) corresponds to interactions between instantons, mediated by oscillations of the plasmon bulk modes, which do not affect the computation of the phase $\gamma$ and will be neglected henceforth. The coupling between the $\vec{\chi}$-modes and the spin degrees of freedom occurs via the boundary Hamiltonian in Eq.(16), which may be presented as

$$
\mathcal{H}_{\mathbf{B}}^{(I)}=\mathcal{B}[\vec{\chi}(\tau)] \cdot \mathbf{S}_{G}(\tau)+B_{0}[\vec{\chi}(\tau)]
$$

with

$$
\begin{aligned}
& B_{0}[\vec{\chi}(\tau)]=2 E_{1} \sum_{j=1}^{3} \cos \left[\vec{\alpha}_{j} \cdot \vec{\chi}(\tau)\right], \\
& \mathcal{B}_{x}[\vec{\chi}(\tau)]=2 E_{3} \sum_{j=1}^{3} \cos \left[\frac{2 \pi}{3}(j-2)\right] \cos \left[\vec{\alpha}_{j} \cdot \vec{\chi}(\tau)\right] \\
& \mathcal{B}_{y}[\vec{\chi}(\tau)]=2 E_{3} \sum_{j=1}^{3} \sin \left[\frac{2 \pi}{3}(j-2)\right] \cos \left[\vec{\alpha}_{j} \cdot \vec{\chi}(\tau)\right] \\
& \mathcal{B}_{z}[\vec{\chi}(\tau)]=2 E_{z} \sum_{j=1}^{3} \cos \left[\vec{\alpha}_{j} \cdot \vec{\chi}(\tau)+\frac{\pi}{2}\right]+B_{\|} .
\end{aligned}
$$

Focusing on the "threefold degenerate" point obtained when $\left(\mu_{1}^{*} \cdot \mu_{2}^{*}\right)=\left(\frac{4 \pi}{3}, 0\right)$, one easily realizes that the instanton trajectories of interest lie along the sides of the triangle whose vertices coincide with the $R, G, B$ points defined by $n_{12}=n_{13}=0$. At the $R, G, B$-vertices one gets

$$
\vec{\chi}_{R}=2 \pi(1,0) \quad, \quad \vec{\chi}_{G}=2 \pi\left(\frac{1}{2},-\frac{1}{\sqrt{3}}\right) \quad, \quad \vec{\chi}_{B}=2 \pi\left(\frac{1}{2}, \frac{1}{\sqrt{3}}\right) .
$$


Any instanton path runs between two of the points in the $\vec{\chi}$-configuration space listed in Eq.(D.5). Moreover, due to

$$
\vec{\alpha}_{2}=\mathcal{R}\left(\frac{2 \pi}{3}\right) \vec{\alpha}_{1} \quad, \quad \vec{\alpha}_{3}=\mathcal{R}\left(\frac{4 \pi}{3}\right) \vec{\alpha}_{1},
$$

with

$$
\mathcal{R}(\theta)=\left[\begin{array}{cc}
\cos (\theta) & \sin (\theta) \\
-\sin (\theta) & \cos (\theta)
\end{array}\right],
$$

one sees that the path connecting $B$ to $R$ may be obtained by acting with $\mathcal{R}\left(\frac{2 \pi}{3}\right)$ on the one connecting $G$ to $B$; in addition, the path connecting $R$ to $G$ may be obtained by acting with $\mathcal{R}\left(\frac{4 \pi}{3}\right)$ on the path connecting $G$ to $B$. As a result, it is enough to compute only one tunnelling amplitude, for example the one between $G$ and $B$. To do so, let us parameterize such an instanton path as $\left(\chi_{1}(\tau), \chi_{2}(\tau)\right)=\left(\pi, \frac{2 \pi}{\sqrt{3}} \sigma(\tau)\right)$, with $\sigma(0)=-\frac{1}{2}$, and $\sigma(\beta)=\frac{1}{2}$. The corresponding Euclidean action is then given by

$$
S[\sigma]=\int_{0}^{\beta} d \tau\left\{\frac{M \pi^{2}}{6}\left[(\dot{\sigma})^{2}+\omega^{2} \sigma^{2}\right]-2 E_{1}-2 E_{3} \sqrt{4-3 \sin ^{2}(\pi \sigma)}\right\} .
$$

The term $\propto \sigma^{2}$ is basically constant, along the instanton path. Thus, the actual value of $S[\sigma]$ may, in principle, be computed by determining the zero-action solution in the "inverted potential", $\sigma_{\text {Inst }}(\tau)$, as

$$
\frac{M \pi^{2}}{6} \dot{\sigma}_{\text {Inst }}^{2}(\tau) \approx 2 E_{3}\left(\sqrt{4-3 \sin ^{2}\left(\pi \sigma_{\text {Inst }}(\tau)\right)}+1\right),
$$

and, then, evaluating $\bar{S}=S\left[\sigma_{\text {Inst }}\right]$. Since the fugacity $Y=e^{-\bar{S}}$ is strongly renormalized by the interaction with the collective plasmon modes of the bulk, it eventually scales as $Y(L) \sim L^{-\frac{g}{3}}$ and, thus, one gets that $e^{-\bar{S}} \sim Y\left(L=L_{0}\right)$.

The tunnelling amplitudes have also a phase stemming from the topological term due to the spin [39]. To derive it, one may consider that the probability amplitude to remain in the same state (for example R), is not only given by the instanton/anti-instanton contributions, but also by the loops around the three degenerate states. The action of such a loop will induce an extra topological term arising from the trajectory of the spin state during the loop. For a generic spin state $|\alpha(\tau)\rangle=\cos \frac{\theta(\tau)}{2}|\Uparrow\rangle+e^{i \phi(\tau)} \sin \frac{\theta(\tau)}{2}|\Downarrow\rangle$ the topological term can be written as:

$$
S_{\text {Top }}=\frac{i}{2} \int_{0}^{T} d \tau \dot{\phi}(\tau)(1+\cos \theta(\tau)) .
$$

Thus the Euclidean action for a loop will be given by:

$$
S_{\text {loop }}[\vec{\chi}(\tau) ; \phi(\tau) ; \theta(\tau)]=S_{\text {Top }}[\phi, \theta]+3 S_{\text {inst }} .
$$

Writing the spin states along the instanton paths and substituting the instanton coordinates $\vec{\chi}(\tau)$ into Eq.(45), one finds the corresponding lowest energy eigenstate. For small $B_{\|}$, one obtains:

$$
|\alpha(\tau)\rangle=\cos \frac{\theta_{f}}{2}|\Uparrow\rangle+e^{i \gamma(\tau)} \sin \frac{\theta_{f}}{2}|\Downarrow\rangle
$$


where $\theta_{f}$ is defined in Eq. (51) and $\gamma(\tau)=\arg \left[B_{x}[\vec{\chi}(\tau)]+I B_{y}[\vec{\chi}(\tau)]\right]$. Since $\theta$ remains constant, the evaluation of $S_{\text {Top }}$ yields

$$
S_{\text {Top }}=i \pi\left(1+\cos \theta_{f}\right) \approx i \pi\left(1-\frac{B_{\|}}{4}\right),
$$

that is, the phase contribution to the amplitude of loop tunnelling. Assuming that the three instanton tunnelling are equivalent, one naturally assign to each tunnelling amplitude a third of the total phase. Thus, the single instanton tunnelling amplitude is given by:

$$
\mathcal{Y}=Y e^{i \frac{\pi}{3}-i \frac{B_{\|}}{12}}
$$

which is the result used in the paper.

\section{References}

[1] I. Affleck, Quantum impurity problems in condensed matter physics, in: Exact Methods in Low-dimensional Statistical Physics and Quantum Computing: Lecture Notes of the Les Houches Summer School: Volume 89, July 2008, arXiv:0809.3474, Oxford Univ Pr, 2010, p. 3.

[2] H. van Houten, C. Beenakker, Quantum point contacts, Physics Today 49 (7) (1996) $22-27$. doi: $10.1063 / 1.881503$.

[3] C. Beenakker, H. van Houten, Quantum point contacts, in: W. Kirk, M. Reed (Eds.), Nanostructures and mesoscopic systems: proceedings of the international symposium, Santa Fe, New Mexico, May 20-24, 1991, Academic Press, 1992.

[4] C. Chamon, M. Oshikawa, I. Affleck, Junctions of three quantum wires and the dissipative hofstadter model, Phys. Rev. Lett. 91 (2003) 206403. arXiv: cond-mat/0305121, doi:10.1103/PhysRevLett.91.206403.

[5] M. Oshikawa, C. Chamon, I. Affleck, Junctions of three quantum wires, J. Stat. Mech. 0602 (2006) P008. arXiv: cond-mat/0509675.

[6] D. Giuliano, P. Sodano, Y-junction of superconducting josephson chains, Nuclear Physics, Section B 811 (3) (2009) 395-419. doi : http://dx .doi.org/10.1016/j.nuclphysb.2008.11.011.

[7] J. Cardy, Boundary conformal field theory, Encyclopedia of mathematical physics, arXiv:hep-th/0411189v2.

[8] T. Giamarchi, Quantum physics in one dimension, Oxford University Press, USA, 2004.

[9] A. M. Tsvelick, P. B. Wiegmann, Exact results in the theory of magnetic alloys, Advances in Physics 32 (1983) $453-713$.

[10] P. Schlottmann, Some exact results for dilute mixed-valent and heavy-fermion systems, Physics Reports 181 (12) (1989) 1 - 119. doi : 10.1016/0370-1573(89)90116-6.

[11] P. Nozières, A. Blandin, Kondo effect in real metals, J. Phys. France 41 (3) (1980) $193-211$. doi: 10.1051/jphys:01980004103019300.

[12] I. Affleck, A. Ludwig, Exact conformal-field-theory results on the multichannel kondo effect: Single-fermion greens function, self-energy, and resistivity, Physical Review B 48 (10) (1993) 7297-7321.

[13] P. Fendley, A. Ludwig, H. Saleur, Exact conductance through point contacts in the $v=1 / 3$ fractional quantum hall effect, Physical review letters 74 (15) (1995) 3005-3008.

[14] A. M. Chang, Chiral luttinger liquids at the fractional quantum hall edge, Rev. Mod. Phys. 75 (4) (2003) 14491505. doi:10.1103/RevModPhys.75.1449.

[15] C. L. Kane, M. P. A. Fisher, Transmission through barriers and resonant tunneling in an interacting onedimensional electron gas, Physical Review B 46 (23) (1992) 15233-15262.

[16] C. L. Kane, M. P. A. Fisher, Transport in a one-channel luttinger liquid, Phys. Rev. Lett. 68 (8) (1992) 12201223. doi:10.1103/PhysRevLett.68.1220.

[17] S. A. Reyes, A. M. Tsvelik, Crossed spin-1/2 heisenberg chains as a quantum impurity problem, Phys. Rev. Lett. 95 (18) (2005) 186404. doi : 10.1103/PhysRevLett.95.186404.

[18] D. Giuliano, P. Sodano, Effective boundary field theory for a josephson junction chain with a weak link, Nucl. Phys. B 711 (3) (2005) 480-504. doi : http: //dx.doi.org/10.1016/j.nuclphysb.2005.01.037. 
[19] D. Giuliano, P. Sodano, Boundary field theory approach to the renormalization of squid devices, Nucl. Phys. B 770 (3) (2007) 332-370. doi : http: //dx .doi.org/10.1016/j.nuclphysb.2007.02 .015.

[20] D. Giuliano, P. Sodano, Pairing of cooper pairs in a josephson junction network containing an impurity, Europhysics Letters 88 (2009) 17012. doi : http://dx.doi.org/10.1209/0295-5075/88/17012.

[21] D. Giuliano, P. Sodano, Competing boundary interactions in a josephson junction network with an impurity, Nuclear Physics B 837 (3) (2010) 153 - 185. doi:10.1016/j .nuclphysb.2010.04.022.

[22] L. Glazman, A. Larkin, New quantum phase in a one-dimensional josephson array, Phys. Rev. Lett. 79 (19) (1997) 3736-3739. doi : doi : 10.1103/PhysRevLett.79.3736.

[23] F. Hekking, L. Glazman, Quantum fluctuations in the equilibrium state of a thin superconducting loop, Phys. Rev. B 55 (10) (1997) 6551-6558. doi : 10.1103/PhysRevB.55.6551.

[24] D. Giuliano, P. Sodano, Frustration of decoherence in y-shaped superconducting josephson networks, New Journal of Physics 10 (2008) 093023. doi : http://dx. doi .org/10.1088/1367-2630/10/9/093023.

[25] Y. Makhlin, G. Schön, A. Shnirman, Quantum-state engineering with josephson-junction devices, Rev. Mod. Phys. 73 (2) (2001) 357-400. doi:10.1103/RevModPhys.73.357.

[26] M. V. Feigel'man, L. B. Ioffe, V. B. Geshkenbein, P. Dayal, G. Blatter, Superconducting tetrahedral quantum bits, Phys. Rev. Lett. 92 (9) (2004) 098301. doi :10.1103/PhysRevLett.92.098301.

[27] M. V. Feigel'man, L. B. Ioffe, V. B. Geshkenbein, P. Dayal, G. Blatter, Superconducting tetrahedral quantum bits: Emulation of a noise-resistant spin- 12 system, Phys. Rev. B 70 (22) (2004) 224524. doi:10.1103/PhysRevB.70.224524.

[28] R. B. Usmanov, L. B. Ioffe, Theoretical investigation of a protected quantum bit in a small Josephson junction array with tetrahedral symmetry, Phys. Rev. B 69 (21) (2004) 214513. doi : 10.1103/PhysRevB.69.214513.

[29] C. van der Wal, F. Wilhelm, C. Harmans, J. Mooij, Engineering decoherence in josephson persistent-current qubits, The European Physical Journal B - Condensed Matter and Complex Systems 31 (2003) 111-124. doi:10.1140/epjb/e2003-00015-9.

[30] H. J. Shulz, G. Cuniberti, P. Pieri, Fermi liquids and luttinger liquids, in: Morandi et al. [40].

[31] C. N. Yang, C. P. Yang, One-dimensional chain of anisotropic spin-spin interactions. ii. properties of the ground-state energy per lattice site for an infinite system, Phys. Rev. 150 (1) (1966) 327-339. doi:10.1103/PhysRev.150.327.

[32] P. Agren, K. Andersson, D. B. Haviland, Kinetic inductance and coulomb blockade in one dimensional josephson junction arrays, Journal of Low Temperature Physics 124 (2001) 291-304. doi:10.1023/A:1017594322332.

[33] J. Cardy, Scaling and renormalization in statistical physics, Cambridge Univ Pr, 1996.

[34] J. Cardy, Conformal invariance and surface critical behavior, Nuclear Physics B 240 (4) (1984) 514-532.

[35] G. Yuval, P. W. Anderson, Exact results for the kondo problem: One-body theory and extension to finite temperature, Phys. Rev. B 1 (4) (1970) 1522-1528. doi : 10.1103/PhysRevB.1.1522.

[36] P. Goswami, S. Chakravarty, Dissipation, topology, and quantum phase transition in a one-dimensional josephson junction array, Phys. Rev. B 73 (9) (2006) 094516. doi : 10.1103/PhysRevB .73.094516.

[37] S. Coleman, Aspects of symmetry: selected Erice lectures of Sidney Coleman, Cambridge Univ Pr, 1988, Ch. Chap. 7, The Uses of Instantons.

[38] A. Auerbach, F. Berruto, L. Capriotti, Quantum magnetism approaches to strongly correlated electrons, in: Morandi et al. [40].

[39] F. Wilczek, A. Shapere, Geometric phases in physics, World Scientific Pub Co Inc, 1989.

[40] G. Morandi, P. Sodano, V. Tognetti, A. Tagliacozzo (Eds.), Field Theories for Low-Dimensional Condensed Matter Systems: Spin Systems and Strongly Correlated Electrons, Springer, 2000. 